\title{
The bending of fully nonlinear beams. Theoretical, numerical and experimental analyses
}

\begin{abstract}
This paper deals with the equilibrium problem of fully nonlinear beams in bending by extending the model for the anticlastic flexion of solids recently proposed by L. Lanzoni and A.M. Tarantino [1] in the context of finite elasticity. In the first part of the paper it is shown, through a parametric analysis, that some geometrical parameters of the displacement field lose importance when slender beams are considered. Therefore, kinematics is reformulated and, subsequently, a fully nonlinear theory for the bending of slender beams is developed. In detail, no hypothesis of smallness is introduced for the deformation and displacement fields, the constitutive law is considered nonlinear and the equilibrium is imposed in the deformed configuration. Explicit formulas are obtained which describe the displacement fields of the inflexed beam, the stretches and the stresses for each point of the beam using both the Lagrangian and Eulerian descriptions. All these formulas are linearized by retrieving the classical formulae of the infinitesimal bending theory of beams. In the second part of the paper the theoretical results are compared with those provided by numerical and experimental analyses developed for the same equilibrium problem with the aim of justify the hypotheses underlying the theoretical model. The numerical model is based on the finite element method (FEM), whereas a test equipment prototype is designed and manufactured for the experimental analysis.
\end{abstract}

Keywords: Finite elasticity; Hyperelasticity; Equilibrium; Beam; Bending moment; Anticlastic curvature; Experimental pure-bending.

Preprint submitted to Journal of ${ }^{A} T_{E} X$ Templates

August 26, 2019 


\section{Introduction}

2 The flexure of nonlinear beams has been widely investigated because of its great relevance in many technical applications. Still today, however, a fully non-

4 linear analysis of this classic problem seems to escape to a convincing modelling. This is basically due to the nonlinearities involved in the problem formulation,

6 such as those related to constitutive laws and to the impossibility of using the smallness hypothesis for both the displacement field (which allows to impose the equilibrium conditions in the undeformed configuration) and the displacement gradient field (which permits to adopt linearized deformation measures). Even

10 when all nonlinearities are taken into account, as in the few cases developed in the context of the finite elasticity, the complexity is such that only models that describe approximately the behaviour of a body inflexed have been proposed in Literature.

One of the first investigations in the framework of finite elasticity was carried out by Seth [2], who studied a plate under flexure in the absence of body forces.

${ }_{16}$ Based on the semi-inverse method, he assumed the deformed configuration of the plate like a circular cylindrical shell, keeping valid the Bernoulli-Navier

18 hypothesis for cross sections. Moreover, he assumed that the stress depends on the strain according to the linearized theory of elasticity. In his work, the bending couples needed to induce the hypothesized configuration of the plate together with the position of the unstretched fibre within the plate thickness 22 (neutral axis) were also assessed.

The flexion problem of an elastic block was extended by Rivlin [3], using a stored energy function for incompressible materials according to Mooney. The deformation considered by Rivlin transforms the elastic block into a short cylinder with the base having the shape of a circular crown sector. No displacements along the axis of the cylinder were taken into account, making the problem

${ }_{28}$ as a matter of fact two-dimensional. Surface traction necessary to induce the assumed displacement field was determined, showing that in the case of a Neo- 
posite couples acting at the end faces.

Other contributions, always based on Rivlin solution, were proposed by Ericksen [4, Carroll [5, Wang [6] and Aron and Wang [7. Universal results in finite elasticity for the bending of a rectangular parallelepiped into a cylindrical annular sector are listed in review paper by Saccomandi [8].

36

Shield 9] studied the problem of the bending of a beam by assuming small strains but large displacements. He retrieved the linear Lamb solution [10] for

38 the deflection of the middle surface of the beam. As remarked in this work, for large values of the width-to-thickness ratio, the deflection profile is flat in the central portion of the cross section and oscillatory near the edges.

All the aforementioned works address the bending problem in a two-dimensional 42 context, systematically neglecting the pure deformation of the cross sections. In this way, the modelling of the problem was substantially simplified, since the 44 displacement field is assumed to be plane.

A wide amount of studies dealing the flexion with large deflections of beams, 46 under several loading and clamping conditions, can be found in the Literature. A significant part of these studies was based on the solution of the Elastica 48 according to the well-known Euler-Bernoulli law for bending ( $c f$. Love [11]). After some studies about a cantilever beam subjected at its free edge to a con50 centrated vertical load, Wang et al. [12] proposed a straightforward numerical approach to solve the equilibrium problem of beams under different load distri${ }_{52}$ butions. On the same subject, other contributions were provided by Wang [13] and Holden 14. A comprehensive review on applications of the Elastica can be

${ }_{54}$ found in the book by Frisch-Fay [15. In all these works, a linear law between the curvature and the bending moment was assumed.

56 Likewise to the Elastica, Reissner [16] modelled a beam as an inextensible one-dimensional system considering in addition the shearing deformation. Using 58 the principle of virtual works, he derived a nonlinear equilibrium equation for beams and subsequently extended the analysis to thin curved beams [17, 18.

60

In the framework of finite elements method (FEM) for nonlinear analysis of structures, many works concerning the large displacements and large rotations 
of beams were carried out. As an example, Bathe and Bolourchi [19] reported both a total Lagrangian formulation and an updated Lagrangian formulation to perform FEM analyses suitable for 3D beams. Both formulations were based on incremental equilibrium equations and proper decomposition of stresses and 66 strains. Cubic interpolating functions were assumed to describe the displacement field related to bending. This study shown that the updated Lagrangian

${ }_{68}$ formulation is computationally effective, as confirmed by a number of sample solutions about shallow arches and cantilever beams, including the case of an 70 initially curved beam. Although both displacements and rotations were considered large in this reference, the strains were assumed to be small.

72 A straightforward parametrization of the equation of motion suitable for FEM formulation of beams was proposed by Simo [20. In that work, the con74 figuration of a beam was completely described by an orthogonal matrix, from which both the rigid rotations of cross sections and the position of the centroids 76 can be inferred. In addition, it was shown that the formulation reported by Reissner [16] is exactly retrieved when a plane problem is considered.

78 In the works by Cardona and Geradin [21] and by Simo and Vu-Quoc [22, a vector description of rotations was assumed and the parametrization of finite rotations was discussed in detail. The governing equations were derived by assuming that cross sections do not change and preserve their planarity during 82 the deformation process. The motion equations of beams were made explicit both in spatial and material settings. For both static and dynamic situations

84 a FEM analysis was performed. Following this approach other contributions are given in 23. However, in these works, neglecting the quadratic part of the

${ }_{86}$ Green-Lagrange strain tensor, small strains were considered and in addition a linear constitutive relation was adopted.

$88 \quad$ Moreover, in all these works concerning beams under finite displacements [19, 23] (as well as in the case of the Elastica [11]-[18] or in the case of models 90 developed in the context of the finite elasticity [2]-9]), the pure deformation of cross sections was completely neglected, because during deformation these ${ }_{92}$ maintain their shape and size, changing rigidly just the position. On the con- 
trary, according to a more adherent view to physical behaviour of beams, the

pure deformation of cross sections should always be taken into consideration, since the longitudinal inflexion is always coupled to the transversal one. More specifically, also cross sections are inflexed with a curvature which is opposite to that longitudinal, and the two curvatures have comparable magnitudes. The transversal deformation just described is known in Literature as anticlastic effect.

In this regard, it should be kept in mind that the pure deformation of the cross sections is usually taken into account even in the classic linear bending theory of beams.

Recently Lanzoni and Tarantino [1] proposed a fully nonlinear analysis of solids under anticlastic bending. In [1], a three-dimensional kinematic model, where the longitudinal bending is accompanied by the transversal deformation of cross sections, is formulated. By following a semi-inverse approach, the displacement field, containing some free unknown parameters, is obtained. Successively, through the equilibrium equations and the boundary conditions, these free parameters are determined. The current paper continues this study, addressing specifically and developing further the analysis for beams, namely for the particular case of solids with a predominant dimension on the other two.

Section 2 investigates the theoretical model. In particular, in Section 2.1, the displacement field obtained in [1] and the constitutive law adopted are recalled. ${ }_{14}$ In Section 2.2 the equilibrium equations in dimensionless form are derived. By varying the geometrical and constitutive parameters, it was shown by means of 16 a numerical analysis that these equilibrium equations are substantially satisfied at each point of the beam. Furthermore, this preliminary study shows that, as the beam becomes more and more slender, some geometrical quantities of the displacement field lose their physical meaning. Based on these observations, the kinematics is reformulated in Section 2.3 and a fully nonlinear theory is developed for the class of slender beams. Explicit formulas for stretches, Piola22 Kirchhoff and Cauchy stresses are provided. The nonlinear bending theory is linearized in Section 2.4 by introducing the hypothesis of smallness for both 


\subsection{Displacement field}

\section{The theoretical model}

the deformation and displacement fields and getting the well-known formulas of the linear bending theory of slender beams. The results provided by the theoretical model are then compared in Section 3 with the results obtained by the numerical and experimental analyses. In Section 3.1, the numerical model developed for the bending of nonlinear beams, using the finite element method (FEM), is presented and discussed in detail. The experimental apparatus specially designed and constructed for the large bending of beams is described in Section 3.2. The experimental results are provided in terms of DIC monitoring, i.e. the full-field optical monitoring technique. Displacement fields evaluated with the theoretical model, the FE model and the experimental tests are compared to each other in Section 3.3. Furthermore, particular attention is paid to the evaluation of the anticlastic radius and to its variability inside of the cross section as well as to estimate the reliability of the hypothesis of conservation of planarity of the cross sections. Finally, conclusions are drawn in Section 4.

In this Section, some authors' results for the finite anticlastic bending of solids [1] are recalled and at the same time the notation is introduced.

Let us consider a hyperelastic beam $\overline{\mathcal{B}}$ composed of a homogeneous, isotropic and compressible material, having the shape of a rectangular parallelepiped.

${ }_{144}$ The width, height and length of this parallelepiped are denoted by $B, H$ and $L$, respectively. Reference is made to a Cartesian coordinate system $\{O, X, Y, Z\}$

${ }_{146}$ having the origin $O$ placed in the centroid of the beam, as shown in Figure 1 Although the formulation will be developed for a beam with a rectangular cross section, it can be readily extended to beams with a generic cross section provided that the symmetry with respect to the $Y$ axis is maintained.

The undeformed configuration $\overline{\mathcal{B}}$ of the beam is assumed as the reference configuration, whereas the deformed configuration is given by the deformation 


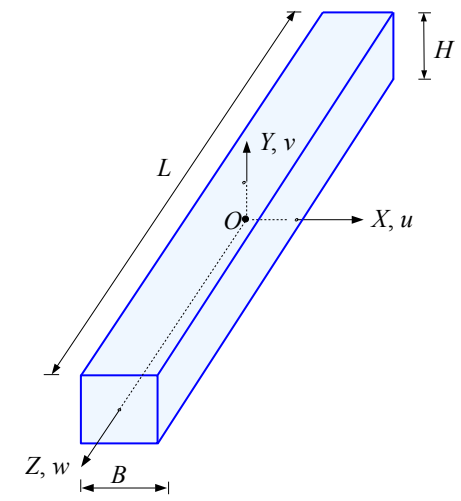

Figure 1: Prismatic beam $\overline{\mathcal{B}}$.

$\mathbf{f}: \overline{\mathcal{B}} \rightarrow \mathcal{V}$, Th that is a smooth enough, injective and orientation-preserving (in the sense that det $\mathbf{D f}>0$ ) vector field. The deformation of a generic material point $P$ can be expressed by the well-known relationship

$$
\mathbf{f}(P)=\mathbf{s}(P)+\mathbf{i d}(P)
$$

where $\mathbf{i d}(P)$ and

$$
\mathbf{s}(P)=u(P) \mathbf{i}+v(P) \mathbf{j}+w(P) \mathbf{k},
$$

are the position and displacement vectors of the point $P$. Into (2), the functions $u(P), v(P)$ and $w(P)$ are the scalar components of $\boldsymbol{s}(P)$, whereas $\mathbf{i}, \mathbf{j}$ and $\mathbf{k}$ ${ }_{158}$ denote the unit vectors. The application of the material gradient operator $\mathbf{D}(\cdot)$ to (1) gives

$$
\mathbf{F}=\mathbf{H}+\mathbf{I}
$$
gradients, respectively $2^{2} \mathbf{I}$ is the identity tensor. Points belonging to the de$w$ are referred to the reference system $\{O, X, Y, Z\}$. Fixed notation, we now move to the description of the displacement field.

\footnotetext{
${ }^{1} \mathcal{V}$ is the vector space associated with the three-dimensional Euclidean space $\mathcal{E}$.

${ }^{2} \mathrm{Lin}$ is the set of all (second order) tensors whereas $\mathrm{Lin}^{+}$is the subset of tensors with positive determinant.
} 
In order to determine the displacement field of a nonlinear beam longitudi-

nally inflexed, the following basic hypotheses are introduced.

1. The beam is inflexed longitudinally with constant curvature. Namely, each rectilinear segment of the beam, parallel to the $Z$ axis, is transformed into an arc of circumference.

2. Plane cross sections, orthogonal to the $Z$ axis, remain as such after the beam has been inflexed. Cross sections can deform only in their own plane and all in the same way.

3. As a result of longitudinal inflexion, the beam is inflexed also transversally. Even this transversal inflexion has constant curvature, in such a way that any horizontal plane of the beam is transformed in a toroidal open surface.

4. Slender beams with compact cross sections are considered.

The longitudinal inflexion can be considered as generated by the application of a pair of self-balanced bending moments or by a geometric boundary condition which imposes a prescribed relative rotation between the two end faces of the beam. For both situations, the first assumption, indispensable in a nonlinear context, requires a uniform inflexion of the beam. The second hypothesis is known as Bernoulli-Navier hypothesis, and it is very popular in the linear mechanics of slender beams under pure bending. This assumption, which predicts the conservation of the planarity of cross sections, provides sufficiently accurate results in the above linear theory, while in our context it is certainly less reliable. In any case, it is expected that such an assumption appears better verified for the central portion of the cross section and less for points close to the boundary, where displacement components out of the plane are predictable. The third hypothesis is clearly approximated, since the transversal curvature in general will not be constant, but it will depend on the position of the material point considered within the cross section. However, these first three hypotheses will be better satisfied in the case of compact cross sections, in which height and width are comparable and when the length of the beam is greater than height and width. Namely, in the geometrical conditions specified by the fourth 
hypothesis.

In their analysis, Lanzoni and Tarantino [1], considering only the first three hypotheses, deduced the following expressions for the components of the displacement field:

$$
\left\{\begin{array}{l}
u=-X+r e^{-\frac{1}{r}(Y+O A)} \sin \frac{X}{r} \\
v=-Y-R-O A+\left\{R+r\left[1-e^{-\frac{1}{r}(Y+O A)} \cos \frac{X}{r}\right]\right\} \cos \frac{Z}{R_{0}} . \\
w=-Z+\left\{R+r\left[1-e^{-\frac{1}{r}(Y+O A)} \cos \frac{X}{r}\right]\right\} \sin \frac{Z}{R_{0}}
\end{array}\right.
$$

This kinematical model is the outcome of coupled effects generated by the longitudinal inflexion and by the transversal deformation of cross sections. In (4), $R_{0}$ denotes the radius of the longitudinal arc that does not change its length, that is, the arc whose stretch is unitary, $\lambda_{Z}=1$ ( $c f$. Figure 2(a)). Such a radius $R_{0}$ can be determined by using the geometric boundary condition which prescribes the angle $\alpha_{0}, R_{0}=L / 2 \alpha_{0}$. ${ }^{3}$ In (4), there are three other geometric quantities: $R, r$ and $O A . R$ and $r$ are the longitudinal and transversal radii of the arc with $\lambda_{X}=\lambda_{Y}=1$ (cf. Figure 2(a), Figure 2(b)), respectively. Given hypothesis 2, $r$ assumes the same value for all cross sections variously inclined. $O A$ indicates the ordinate at the origin of the longitudinal arc with $\lambda_{X}=\lambda_{Y}=1$ (cf. Figure 2(a)). These three kinematic parameters are calculated using the following three coupled expressions [1]:

$$
\begin{aligned}
& r\left[a\left(R_{0}^{2}-R^{2}\right)+2 b R_{0}^{2}+c\left(R_{0}^{2}+R^{2}\right)\right]-2 R\left[a R_{0}^{2}+3 b R_{0}^{2}+c\left(R_{0}^{2}+R^{2}\right)\right]=0, \\
& R_{0}-R=r\left(1-\cos \frac{B}{2 r}\right) \\
& O A=r \ln \left[\cosh \left(\frac{H}{2 r}\right)\right] .
\end{aligned}
$$

Eqn (5) was obtained by imposing the equilibrium at the points belonging to the longitudinal basic line: $X=0, Y=-O A$ and $Z=Z$. The eqn (6) was derived from the boundary conditions requiring that the lateral surface of the body is unloaded. These conditions are not satisfy locally but globally. The third expression (7) was attained on the basis of geometric considerations. The positive constants $a, b$ and $c$ in (5) are the constitutive parameters of the

\footnotetext{
${ }^{3}$ In the sequel, the relationship between this angle $\alpha_{0}$ and the pair of self-balanced bending moment to apply to the end faces of the beam will be found.
} 
stored energy function $\omega$ for compressible Mooney-Rivlin materials $(\delta=\operatorname{det} \mathbf{F}=$ $\left.{ }_{218} \quad \lambda_{X} \lambda_{Y} \lambda_{Z}, I_{3}=\delta^{2}\right)$

$$
\omega\left(I_{1}, I_{2}, I_{3}\right)=a I_{1}+b I_{2}+\Gamma(\delta)
$$

where田

$$
\begin{aligned}
I_{1} & =\|\mathbf{F}\|^{2}=\lambda_{X}^{2}+\lambda_{Y}^{2}+\lambda_{Z}^{2}, \\
I_{2} & =\left\|\mathbf{F}^{\star}\right\|^{2}=\lambda_{X}^{2} \lambda_{Y}^{2}+\lambda_{X}^{2} \lambda_{Z}^{2}+\lambda_{Y}^{2} \lambda_{Z}^{2}, \\
I_{3} & =(\operatorname{det} \mathbf{F})^{2}=\lambda_{X}^{2} \lambda_{Y}^{2} \lambda_{Z}^{2} .
\end{aligned}
$$

${ }_{220}$ In $(8), \Gamma(\delta)$ denotes a convex function that satisfies the growth conditions both as $\delta \rightarrow 0^{+}$and as $\delta \rightarrow+\infty$. For this function the expression proposed by Ciarlet 222 and Geymonat 24 , has been chosen ${ }^{5}$

$$
\Gamma(\delta)=c \delta^{2}-d \ln (\delta)
$$

To ensure that in the absence of deformation the stress vanishes, among the four constants of $\omega$ the following relationship was established [26, 27, 28, 29]:

$$
d=2(a+2 b+c) .
$$

For further details on the displacement field (4), the reader is directly referred

\subsection{Numerical checks of equilibrium equations}

\footnotetext{
${ }^{4}$ The following notations: $\|\mathbf{A}\|=\left(\operatorname{tr} \mathbf{A}^{\mathrm{T}} \mathbf{A}\right)^{1 / 2}$ for the tensor norm in the linear tensor space Lin and $\mathbf{A}^{\star}=(\operatorname{det} \mathbf{A}) \mathbf{A}^{-\mathrm{T}}$ for the cofactor of the tensor $\mathbf{A}$ (if $\mathbf{A}$ is invertible) are used.

${ }^{5}$ For this (as for many others) constitutive models in finite elasticity it must however be observed that they have been proposed on the basis of mathematical requirements and that specific experimental tests, especially under multi-axial load conditions, which can validate them are actually lacking. See for example the predictive incoherences evidenced for the rectilinear shear deformation by Destrade et al. 25].
} 
a)

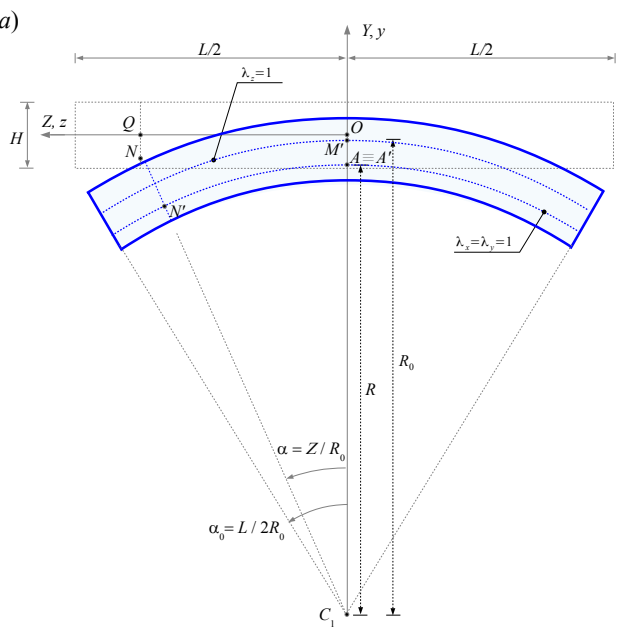

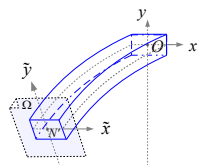

$\alpha$

,

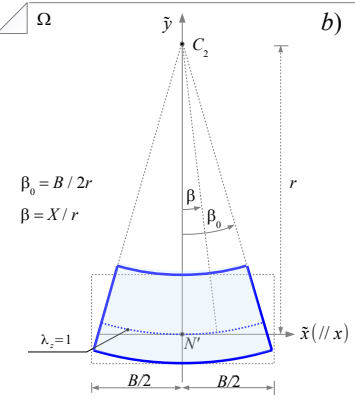

Figure 2: Deformation of the beam. a) Longitudinal deformation in the vertical $Y Z$ plane. b) Transversal deformation in the generic cross section $\Omega$.

tensor that denotes the rotation tensor, whereas $\mathbf{U}$ is a symmetric and positive definite tensor that indicates the right stretch tensor. As known, these two

32 tensors are obtained by the polar decomposition of the deformation gradient $\mathbf{F}=\mathbf{R U}$. Tensor $\mathbf{U}$ is diagonal, because the reference system $\{O, X, Y, Z\}$ 234 is principal for the state of deformation derived from (4). Diagonal components of $\mathbf{U}$ are the stretches, which are also principal. With the derivatives of 236 the displacement field (4), the displacement gradient $\mathbf{H}$ is obtained and then, through (3), the deformation gradient $\mathbf{F}$ is derived. Once known $\mathbf{F}$, the right Cauchy-Green strain tensor $\mathbf{C}$ can be evaluated using its definition. The tensor $\mathbf{C}$ is diagonal and its diagonal components coincide with the squares of the principal stretches. Thus, the following expressions for the principal stretches 
are obtained:

$$
\left\{\begin{array}{l}
\lambda_{X}=\lambda_{Y}=e^{-\frac{1}{r}(Y+O A)} \\
\lambda_{Z}=\frac{R+r\left[1-e^{-\frac{1}{r}(Y+O A)} \cos \frac{X}{r}\right]}{R_{0}}
\end{array}\right.
$$
is

$$
\mathbf{T}_{R}=2\left(\frac{\partial \omega}{\partial I_{1}}+I_{1} \frac{\partial \omega}{\partial I_{2}}\right) \mathbf{F}-2 \frac{\partial \omega}{\partial I_{2}} \mathbf{B F}+2 I_{3} \frac{\partial \omega}{\partial I_{3}} \mathbf{F}^{-\mathrm{T}},
$$

${ }_{250}$ where $\mathbf{T}_{R}$ denotes the (first) Piola-Kirchhoff stress tensor and $\mathbf{B}=\mathbf{F} \mathbf{F}^{\mathrm{T}}$ the left Cauchy-Green strain tensor. Being $\mathbf{B F}=\mathbf{R U}^{3}$ and $\mathbf{F}^{-\mathrm{T}}=\mathbf{R} \mathbf{U}^{-1}$, the above 252

$$
\mathbf{T}_{R}=\mathbf{R S}
$$

Since $\mathbf{U}$ is diagonal ( $c f$. eqn $(13)$ ), even the tensor $\mathbf{S}$ is diagonal

$$
[\mathbf{S}]=\left[\begin{array}{ccc}
\mathrm{S}_{X} & 0 & 0 \\
0 & \mathrm{~S}_{Y} & 0 \\
0 & 0 & \mathrm{~S}_{Z}
\end{array}\right]
$$


254

$$
\mathrm{S}_{J}=2\left(\frac{\partial \omega}{\partial I_{1}}+I_{1} \frac{\partial \omega}{\partial I_{2}}\right) \lambda_{J}-2 \frac{\partial \omega}{\partial I_{2}} \lambda_{J}^{3}+2 I_{3} \frac{\partial \omega}{\partial I_{3}} \frac{1}{\lambda_{J}}, \quad \text { for } J=X, Y, Z
$$

Equilibrium requires that the following vectorial equation must be satisfied 256 locally:

$$
\operatorname{Div} \mathbf{T}_{R}+\mathbf{b}=\mathbf{0}
$$

This vectorial equation, in the absence of body forces $\mathbf{b}$ and after calculating 258 the scalar components of the material divergence of $\mathbf{T}_{R}$, provides a system of three partial differential equations

$$
\left\{\begin{array}{l}
-\left(\frac{\mathrm{S}_{X}}{r}+\mathrm{S}_{Y, Y}\right) \sin \frac{X}{r}+\mathrm{S}_{X, X} \cos \frac{X}{r}=0 \\
{\left[\left(\frac{\mathrm{S}_{X}}{r}+\mathrm{S}_{Y, Y}\right) \cos \frac{X}{r}+\mathrm{S}_{X, X} \sin \frac{X}{r}-\frac{\mathrm{S}_{Z}}{R_{0}}\right] \cos \frac{Z}{R_{0}}-\mathrm{S}_{Z, Z} \sin \frac{Z}{R_{0}}=0} \\
{\left[\left(\frac{\mathrm{S}_{X}}{r}+\mathrm{S}_{Y, Y}\right) \cos \frac{X}{r}+\mathrm{S}_{X, X} \sin \frac{X}{r}-\frac{\mathrm{S}_{Z}}{R_{0}}\right] \sin \frac{Z}{R_{0}}+\mathrm{S}_{Z, Z} \cos \frac{Z}{R_{0}}=0}
\end{array}\right.
$$

where $\mathrm{S}_{J, J}=\frac{\partial \mathrm{S}_{J}}{\partial J}$ for $J=X, Y, Z$ (no sum). The derivatives $\mathrm{S}_{J, J}$ assume the following forms:

$$
\begin{aligned}
\mathrm{S}_{X, X}= & 2\left\{\lambda_{X}\left[\left(\omega_{1, X}+I_{1, X} \omega_{2}+I_{1} \omega_{2, X}\right)-\omega_{2, X} \lambda_{X}^{2}\right]+\frac{I_{3, X} \omega_{3}+I_{3} \omega_{3, X}}{\lambda_{X}}\right\}, \\
\mathrm{S}_{Y, Y}= & 2\left\{\lambda_{Y}\left[\omega_{1, Y}+I_{1, Y} \omega_{2}+\omega_{2, Y}\left(I_{1}-\lambda_{Y}^{2}\right)\right]+\lambda_{Y, Y}\left[\omega_{1}+\omega_{2}\left(I_{1}-3 \lambda_{Y}^{2}\right)\right]\right. \\
& \left.\quad+\frac{1}{\lambda_{Y}}\left[\omega_{3}\left(I_{3, Y}-\frac{I_{3} \lambda_{Y, Y}}{\lambda_{Y}}\right)+I_{3} \omega_{3, Y}\right]\right\}, \\
\mathrm{S}_{Z, Z}= & 0,
\end{aligned}
$$

${ }_{262}$ where $\omega_{i}=\frac{\partial \omega}{\partial I_{i}}$ for $i=1,2,3, I_{i, K}=\frac{\partial I_{i}}{\partial K}$ and $\omega_{i, K}=\frac{\partial}{\partial K}\left(\frac{\partial \omega}{\partial I_{i}}\right)$ for $K=X, Y$, and with

$$
\begin{aligned}
\lambda_{Y, Y} & =\frac{\partial \lambda_{Y}}{\partial Y}=-\frac{e^{-\frac{1}{r}(Y+O A)}}{r} \\
I_{1, X} & =\frac{2 \lambda_{Z}}{R_{0}} e^{-\frac{1}{r}(Y+O A)} \sin \frac{X}{r} \\
I_{1, Y} & =2\left[\frac{\lambda_{Z} e^{-\frac{1}{r}(Y+O A)}}{R_{0}} \cos \frac{X}{r}-\frac{2 e^{-\frac{2}{r}(Y+O A)}}{r}\right] \\
I_{3, X} & =\frac{2 e^{-\frac{5}{r}(Y+O A)} \lambda_{Z}}{R_{0}} \sin \frac{X}{r}, \\
I_{3, Y} & =2 \lambda_{Z}\left[\frac{e^{-\frac{5}{r}(Y+O A)}}{R_{0}} \cos \frac{X}{r}-\frac{2 \lambda_{Z} e^{-\frac{4}{r}(Y+O A)}}{r}\right] .
\end{aligned}
$$


Since $\mathrm{S}_{Z, Z}=0$, the second equation of the system 17 is multiplied by $\cos \frac{Z}{R_{0}}$ and the third equation similarly by $\sin \frac{Z}{R_{0}}$. Now, because the two trigonometric functions $\sin \frac{Z}{R_{0}}$ and $\cos \frac{Z}{R_{0}}$ are never simultaneously zero, system (17) reduces to

$$
\left\{\begin{array}{l}
\mathrm{S}_{X, X} \cos \frac{X}{r}-\left(\frac{\mathrm{S}_{X}}{r}+\mathrm{S}_{Y, Y}\right) \sin \frac{X}{r}=0 \\
\left(\frac{\mathrm{S}_{X}}{r}+\mathrm{S}_{Y, Y}\right) \cos \frac{X}{r}+\mathrm{S}_{X, X} \sin \frac{X}{r}-\frac{\mathrm{S}_{Z}}{R_{0}}=0
\end{array}\right.
$$

where the second equation governs the equilibrium in the $Y$ and $Z$ directions. With the stored energy function (8) for compressible Mooney-Rivlin materials, the following set of derivatives is computed:

$$
\begin{aligned}
& \omega_{1}=a, \quad \omega_{2}=b, \quad \omega_{1, X}=\omega_{1, Y}=\omega_{2, X}=\omega_{2, Y}=0, \\
& \omega_{3}=c-\frac{d}{2 I_{3}}, \quad \omega_{3, X}=\frac{d}{R_{0}} \frac{e^{\frac{3}{r}(Y+O A)}}{\lambda_{Z}^{3}} \sin \frac{X}{r}, \\
& \omega_{3, Y}=-\frac{d}{\lambda_{Z}^{2}} e^{\frac{4}{r}(Y+O A)}\left[\frac{2}{r}-\frac{e^{-\frac{1}{r}(Y+O A)}}{R_{0} \lambda_{Z}} \cos \frac{X}{r}\right] .
\end{aligned}
$$

With these derivatives, the writing of equilibrium eqns 18 , derived from the displacement field (4), is completed. However, system (18) has a rather complex form, which makes its resolution very hard. Moreover, it must be taken in mind that, having been hypothesized a priori the displacement field (4), it does not exist the actual possibility to exactly solve the system for all internal points of the body. Nevertheless, in [1] the existence of a basic longitudinal line, where the equilibrium eqns 18 are fully satisfied, has been recognized. The points belonging to this basic line have the following coordinates: $X=0, Y=-O A$ and $Z=Z$.

When the basic line is abandoned, the equilibrium equations are not fully satisfied. Nevertheless, it is reasonable to expect, as a result of the continuity of the displacement field, that the solution will be yet accurate in a neighbourhood of each single point of the basic line. To show this particular aspect of the problem, in Section 3.1, a specific numerical analysis will be performed to check how much the equilibrium eqns (18), assessed for any point of the cross section, move away from the zero of the basic line. 
With this purpose, in the numerical analysis the geometrical dimensions of

the beam $B, H$ and $L$, the angle of inflexion $\alpha_{0}$ as well as the constitutive parameters $a, b$ and $c$ will be changed, in order to investigate the influence of each parameter. In the following, the adopted units for lengths and forces are $m m$ and $N$, respectively. These variations will be assigned starting from a reference beam, characterized by the following parameters: $B=H=1, L=15$, $\alpha_{0}=\pi / 3, a=1, b=c=0.05$. On the basis of geometrical parameters, the reference beam can be considered as a realistic slender beam, that is a particularly elongated solid where the longitudinal dimension prevails considerably on the two transversal dimensions. The constitutive parameters were chosen to emphasize the term $a$ associated with fibre length variation in the constitutive law, rather than terms $b$ and $c$ corresponding to area and volume changes (see, e.g., [30, 31]), since it plays a more important role in the deformation of a realistic beam.

The geometrical dimensions of the beam, as well as the variable $X, Y$ and $Z$, are normalized by dividing them by $H$. Also the elastic constants are normalized by dividing them by the first constitutive parameter $a$. In the sequel, the same symbols will be used for both normalized and non-normalized parameters. Having made dimensionless the elastic constants also stresses are dimensionless. Consequently, the equilibrium equations become dimensionless and their comparison with the scalar zero takes full meaning.

The results of computations are delivered in two-dimensional diagrams similar to those of Figure 3, where a number of contour lines are shown. These lines join the points where the equilibrium eqns 18 give the same numerical values. A contour-plot highlights the intensity of numerical values. The white colour indicates areas where the eqns $(18)$ are practically met. The intensity of colours grows as the difference from zero increases. Numeric results are given for the particular cross section $Z=0$, but because all cross sections deform in the same way, they are to be considered valid for all. In these figures, the values of the kinematical parameters $r, R$ and $O A$, evaluated by eqns (5/7), are also pointed out. 
a)

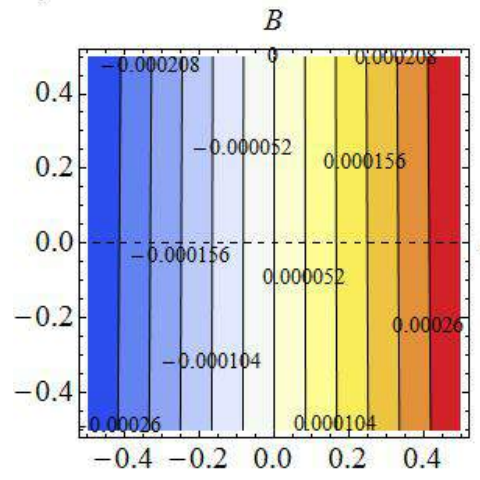

b)

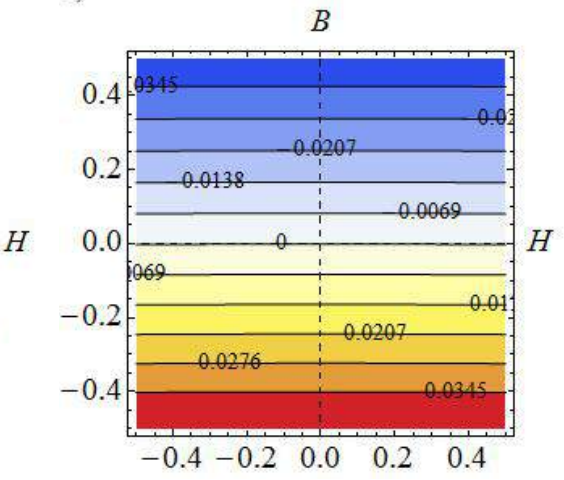

Figure 3: Plot of the equilibrium equations evaluated locally in every point belonging to the cross section $Z=0$. Reference beam. Adopted parameters: $B=1, H=1, L=15, \alpha_{0}=\pi / 3$, $a=1, b=0.05$ and $c=0.05$. Kinematic parameters: $r=89.3399, R=7.1605, O A=$ 0.001399. (a) Eqn 181 , equilibrium along the $X$ axis. (b) Eqn $18{ }_{2}$, equilibrium along the $Y$ and $Z$ axes.

Figure 3(a) shows the numerical results provided by eqn $(18)_{1}$ for the middle cross section $(Z=0)$ of the reference beam. As it can be noted, the numerical values are very close to zero in all points of the cross section and are exactly equal to zero for the vertical middle line $(X=0)$. The Figure $3(\mathrm{~b})$ shows that the second equation of equilibrium $\sqrt{18})_{2}$ is rather well satisfied along a wide horizontal band. Moving toward the upper and lower edges of the cross section, the numerical values gradually increase up to slightly exceed $4 \%$ in these edges. Such a maximum value can be considered in any case small enough and widely acceptable in many technical applications. This small discrepancy can be attributed to the second hypothesis of conservation of the planarity of cross sections and to the third hypothesis that assumes the radius $r$ constant, since these two assumptions are not exactly verified at the edges. As a result, the elimination of this small discrepancy, having to renounce to the second and third hypotheses, may become very complicated.

For this first case examined, with a compact cross section, it can be concluded that exists a large central band, surrounding the longitudinal basic line, where the displacement field (4) can be considered accurate. The solution is a little 
less precise close to the upper and lower edges of the cross section 6

In the following, with reference to this first case shown in Figure 3, the influence exerted by the other parameters will be investigated.

Keeping all the other parameters of the reference beam fixed, Figure 4 shows

a)

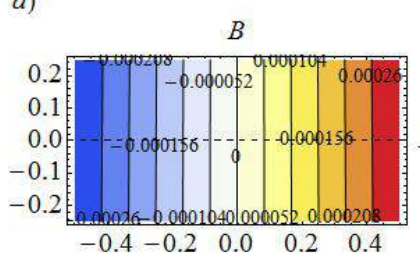

c)

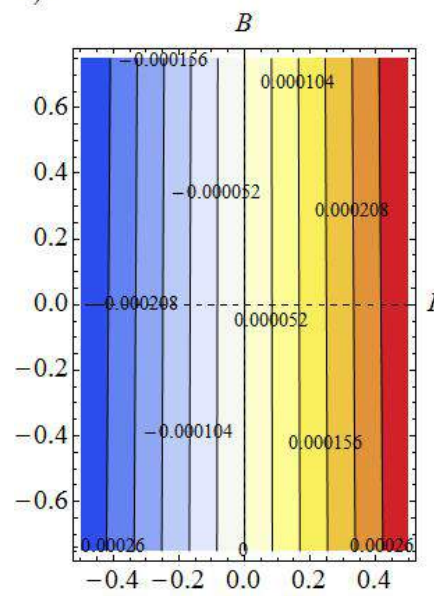

b)

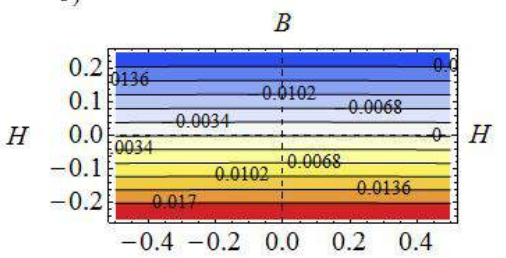

d)

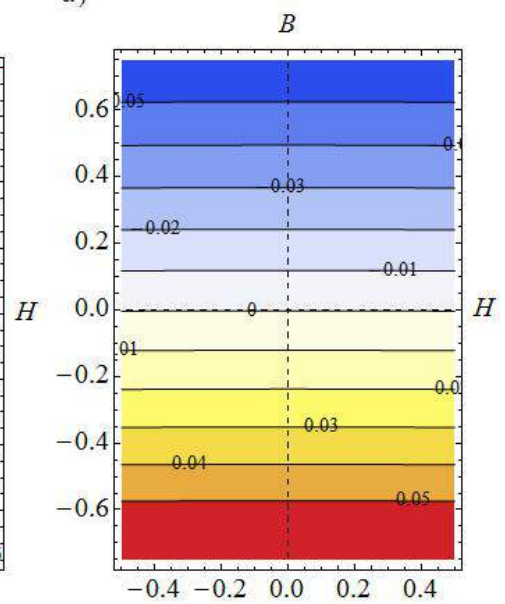

Figure 4: Plot of the equilibrium equations evaluated locally in every point belonging to the cross section $Z=0$. Influence of the geometrical dimension $H$. Adopted parameters: $B=1, L=15, \alpha_{0}=\pi / 3, a=1, b=0.05$ and $c=0.05$. Case with $H=0.5$. Kinematic parameters: $r=89.3399, R=7.1605, O A=0.000349$. (a) Eqn $181_{1}$, equilibrium along the $X$ axis. (b) Eqn $182_{2}$, equilibrium along the $Y$ and $Z$ axes. Case with $H=1.5$. Kinematic parameters: $r=89.3399, R=7.1605, O A=0.003148$. (c) Eqn 181 , equilibrium along the $X$ axis. (d) Eqn $181_{2}$, equilibrium along the $Y$ and $Z$ axes.

two cases in which the height $H$ of the cross section is equal to $H=0.5$ and

\footnotetext{
${ }^{6}$ When it is well identified the physical context in which to apply the model, then, it is possible, in order to estimate specifically the size of such a horizontal band, to define suitable confidence limits for the acceptability of the numerical solution.
} 
$H=1.5$ (in this latter case, the beam still retains a certain degree of slenderness). Figures 4(a) and 4(c) show that in both cases the equilibrium equation in the $X$ direction is practically satisfied. Height $H$ has a greater influence on the equilibrium equations along the $Y$ and $Z$ axes. When $H$ increases, the approximations at the upper and lower edges increase with respect to those of the reference beam ( $c f$. Figure $4(\mathrm{~d})$ ), while the approximations decrease as $H$ decreases ( $c f$. Figure $4(\mathrm{~b})$ ).

Figure 5 shows two cases in which the base $B$ of the cross section is equal to $B=0.5$ and $B=1.5$. The comparison of Figure 5 with Figure 3 , relative to

a)

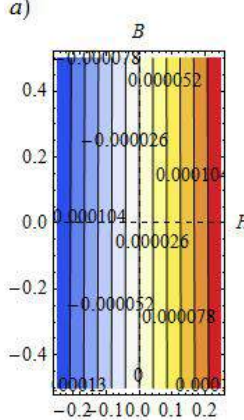

c)

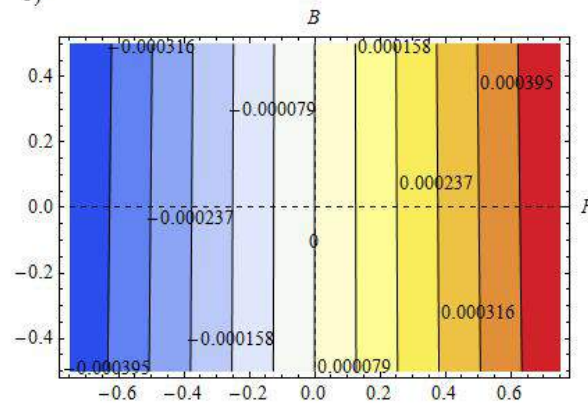

b)

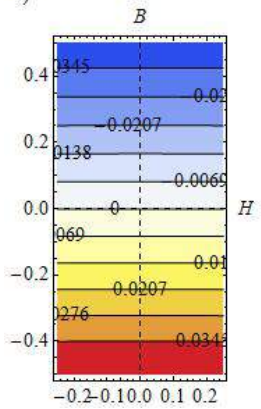

d)

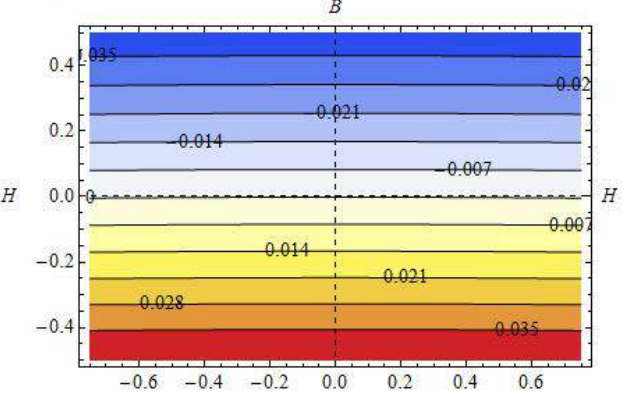

Figure 5: Plot of the equilibrium equations evaluated locally in every point belonging to the cross section $Z=0$. Influence of the geometrical dimension $B$. Adopted parameters: $H=1, L=15, \alpha_{0}=\pi / 3, a=1, b=0.05$ and $c=0.05$. Case with $B=0.5$. Kinematic parameters: $r=89.4785, R=7.1616, O A=0.001396$. (a) Eqn 181 , equilibrium along the $X$ axis. (b) Eqn 18$]_{2}$, equilibrium along the $Y$ and $Z$ axes. Case with $B=1.5$. Kinematic parameters: $r=89.1091, R=7.1588, O A=0.001403$. (c) Eqn 18,1 , equilibrium along the $X$ axis. (d) Eqn $[18]_{2}$, equilibrium along the $Y$ and $Z$ axes. 

an important role on the solution.

Figure 6 shows the influence of length $L$ of the beam. If the length of the

a)

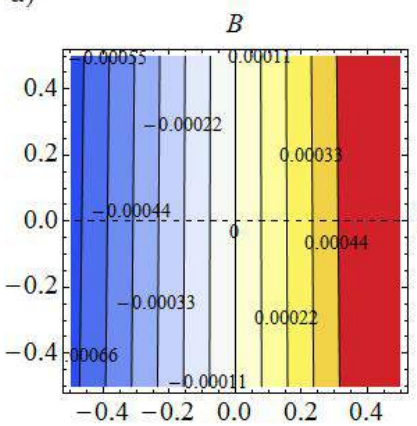

c)

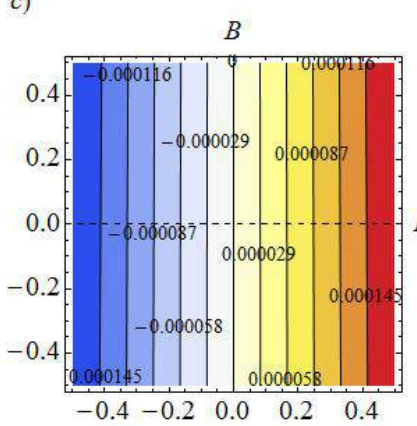

b)

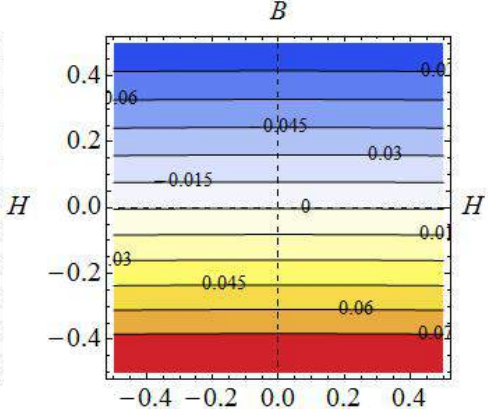

d)

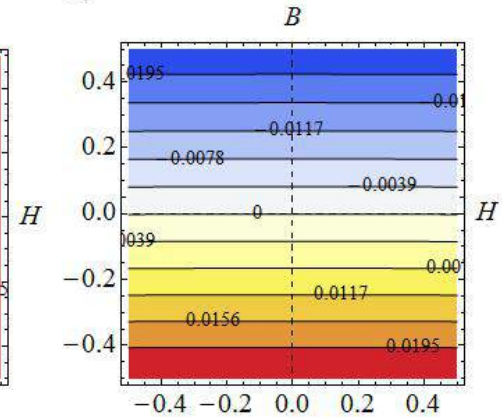

Figure 6: Plot of the equilibrium equations evaluated locally in every point belonging to the cross section $Z=0$. Influence of the length of the beam $L$. Adopted parameters: $H=1, B=1$, $\alpha_{0}=\pi / 3, a=1, b=0.05$ and $c=0.05$. Case with $L=10$. Kinematic parameters: $r=59.4060, R=4.7725, O A=0.002104$. (a) Eqn 181 , equilibrium along the $X$ axis. (b) Eqn $182_{2}$, equilibrium along the $Y$ and $Z$ axes. Case with $L=20$. Kinematic parameters: $r=119.2277, R=9.5482, O A=0.001048$. (c) Eqn $18{ }_{1}$, equilibrium along the $X$ axis. (d) Eqn $182_{2}$, equilibrium along the $Y$ and $Z$ axes.

350

reference beam from $L=15$ is reduced to $L=10$, the errors at the edges are doubled for both equilibrium equations ( $c f$. Figures 6(a) and 6(b)). Instead, if the length of the beam is increased to $L=20$ then there are halved errors at the edges ( $c f$. Figures 6(c) and 6(d)). Therefore the solution becomes more accurate as $L$ increases, that is, in the case of very slender beams. In particular, ${ }^{56}$ for the last case with $L=20$, numerical values given by (18), for all points of 
the cross section, really differ little from the zero of basic line.

The effect of the angle of inflexion $\alpha_{0}$, imposed to the beam by means of the boundary conditions, is illustrated by Figure 7 If the angle $\alpha_{0}$ of the reference beam is halved, reducing it to $\pi / 6$, the values provided by the equilibrium equations become practically zero ( $c f$. Figures $7(\mathrm{a})$ and $7(\mathrm{~b})$ ). Conversely, the approximations increase by increasing the angle $\alpha_{0}$ ( $c f$. Figures $7(\mathrm{c})$ and $7(\mathrm{~d})$ ). In this last case, where the axis of the beam is inflected until to form a semicircle, errors exceed $7 \%$. The foregoing remarks point out that the angle $\alpha_{0}$, as well as the length $L$ of the beam, plays an important role on the accuracy of the solution.

Definitively, the above numerical computations show that in the cross sections there is a central horizontal band, where the numerical solution is acceptable, because close to the exact solution of the longitudinal basic line. Main approximations, due to a non complete vanishing of the equilibrium eqn 18$]_{2}$, remain confined to the upper and lower edges of the cross sections. These errors 32 are generally small. In addition, in the case of very slender beams or in the case of moderate angles $\alpha_{0}$, the proposed model is able to provide very accurate solutions.

Figures 8 and 9 show the influence of constitutive parameters $b$ and $c$.

When the constant $b$ equals the constant $a$, errors have almost doubled (cf. Figure $8(\mathrm{~b})$ ). A similar behaviour occurs also if the constant $c$ grows up to one ${ }_{38}$ (cf. Figure 9(b)). By way of example, for rubber-like materials, the constant $b$ unlikely reaches the unitary value, while the constant $c$ can overcome the unit.

Figure 10 shows the non vanishing components of the Piola-Kirchhoff stress tensor, evaluated in the middle cross section $Z=0$ of the reference beam. For this cross section, the components $\mathrm{T}_{R, 23}, \mathrm{~T}_{R, 31}$ and $\mathrm{T}_{R, 32}$ are zero, $\mathrm{T}_{R, 11}=$ $\mathrm{T}_{R, 22}$ and $\mathrm{T}_{R, 12}=-\mathrm{T}_{R, 21}$. Note that the out-plane component $\mathrm{T}_{R, 33}$ (cf. Figure 10(a)) is much greater than the in-plane components $\mathrm{T}_{R, 11}, \mathrm{~T}_{R, 22}, \mathrm{~T}_{R, 12}$ and $\mathrm{T}_{R, 21}(c f$. Figures 10(b), 10(c) and 10(d)). In fact, these latter are very close to zero in every point of the cross section. At the upper boundary $\mathrm{T}_{R, 33}$ assumes the maximum tensile values and at the lower boundary the maximum 
a)

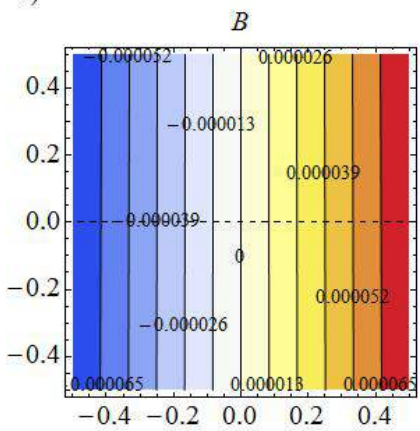

c)

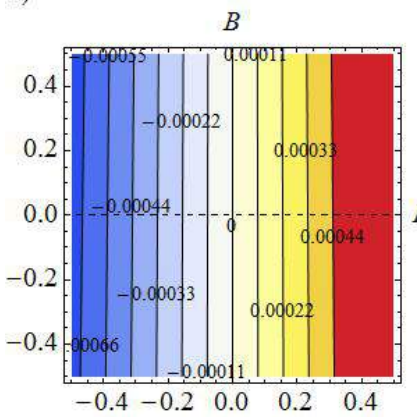

b)

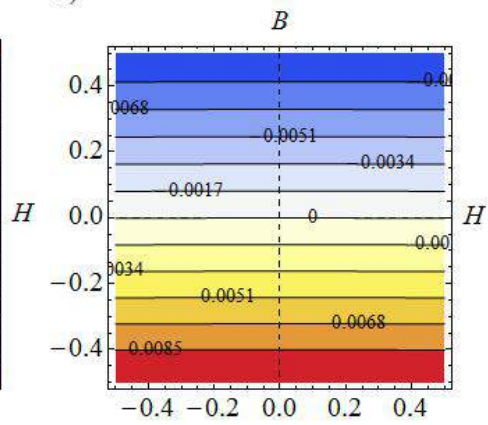

d)

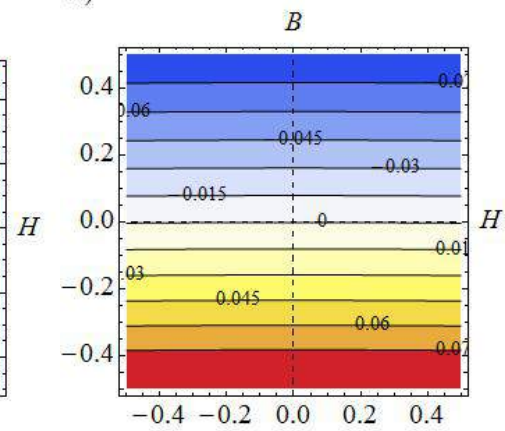

Figure 7: Plot of the equilibrium equations evaluated locally in every point belonging to the cross section $Z=0$. Influence of the angle of inflexion $\alpha_{0}$. Adopted parameters: $B=1, H=$ $1, L=15, a=1, b=0.05$ and $c=0.05$. Case with $\alpha_{0}=\pi / 6$. Kinematic parameters: $r=178.9569, R=14.3232, O A=0.000698$. (a) Eqn 18,1 , equilibrium along the $X$ axis. (b) Eqn $181_{2}$, equilibrium along the $Y$ and $Z$ axes. Case with $\alpha_{0}=\pi / 2$. Kinematic parameters: $r=59.4060, R=4.7725, O A=0.002104$. (c) Eqn $181_{1}$, equilibrium along the $X$ axis. (d) Eqn 18,2 , equilibrium along the $Y$ and $Z$ axes.

compression values. The curve of points with $\mathrm{T}_{R, 33}=0$ is practically a horizontal straight line that passes very close to the centroid of the cross section.

390 Keeping fixed the other parameters, all stress components reduce for $\alpha_{0}$ decreasing and $L$ increasing.

To complete the boundary-value problem that governs the equilibrium of beams, the boundary conditions must be added to the field eqns (18). The boundary conditions on the two beam bases (i.e. the two cross sections with $Z=-L / 2$ and $Z=L / 2$ ) are used to prescribe the angle $\alpha_{0}$. For the lateral 996 surface of the beam, the boundary conditions can be imposed by requiring that 
a)

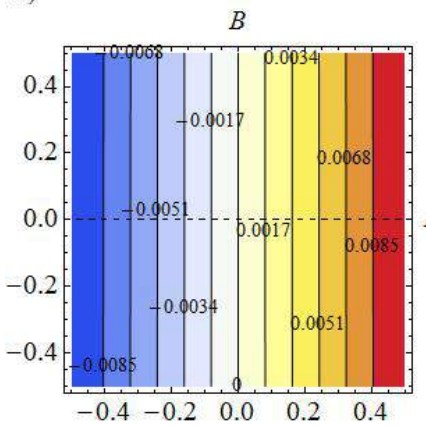

b)

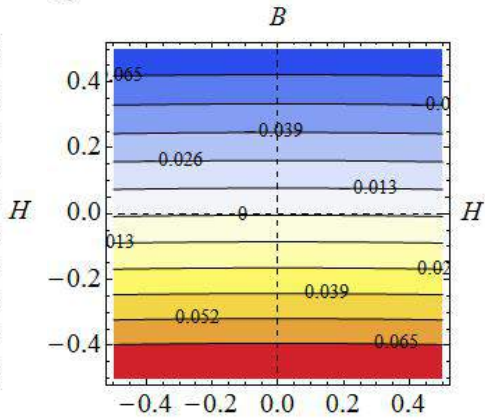

Figure 8: Plot of the equilibrium equations evaluated locally in every point belonging to the cross section $Z=0$. Influence of the constitutive parameter $b$. Adopted parameters: $B=1, H=1, L=15, a=1$ and $c=0.05$. Case with $b=1$. Kinematic parameters: $r=27.9321, R=7.15749, O A=0.0044741$. (a) Eqn 18 , equilibrium along the $X$ axis. (b) Eqn 18,2 , equilibrium along the $Y$ and $Z$ axes.

a)

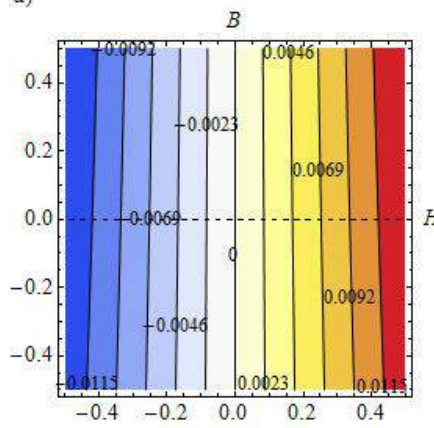

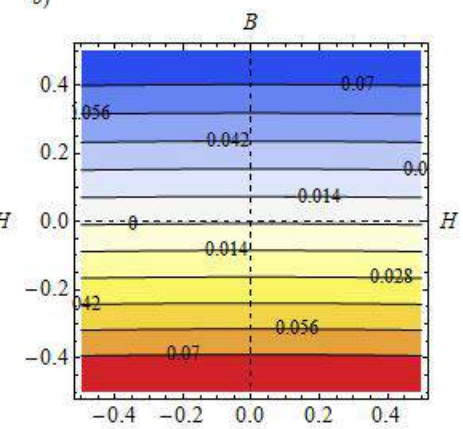

Figure 9: Plot of the equilibrium equations evaluated locally in every point belonging to the cross section $Z=0$. Influence of the constitutive parameter c. Adopted parameters: $B=1, H=1, L=15, a=1$ and $b=0.05$. Case with $c=1$. Kinematic parameters: $r=21.4573, R=7.1561, O A=0.005824$. (a) Eqn 18$]_{1}$, equilibrium along the $X$ axis. (b) Eqn 18,2 , equilibrium along the $Y$ and $Z$ axes.

it is unloaded

$$
\begin{aligned}
\mathbf{t}_{R}=\mathbf{T}_{R} \mathbf{n}=\mathbf{0}, & \text { for } X= \pm \frac{B}{2}, \quad Y \in\left[-\frac{H}{2}, \frac{H}{2}\right], \quad Z \in\left[-\frac{L}{2}, \frac{L}{2}\right], \\
& \text { for } Y= \pm \frac{H}{2}, \quad X \in\left[-\frac{B}{2}, \frac{B}{2}\right], \quad Z \in\left[-\frac{L}{2}, \frac{L}{2}\right],
\end{aligned}
$$

where $\mathbf{t}_{R}$ is the Piola-Kirchhoff stress vector and $\mathbf{n}$ is the outward unit normal. 

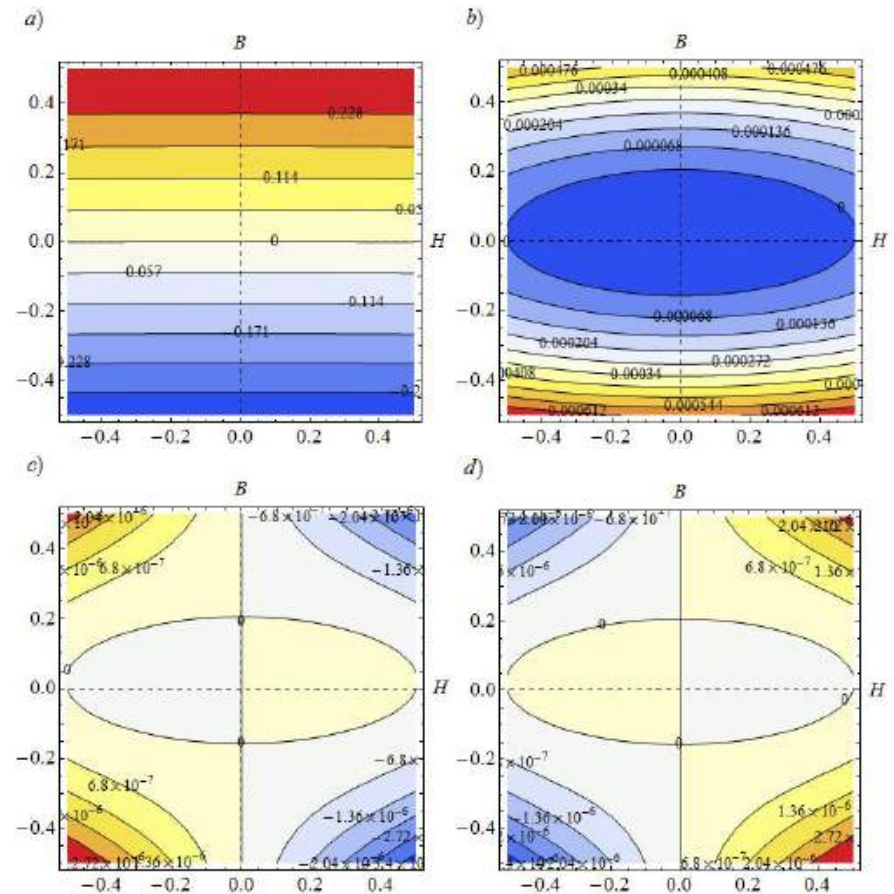

Figure 10: Plot of the components of the Piola-Kirchhoff stress tensor evaluated locally in every point belonging to the cross section $Z=0$. Adopted parameters: $B=1, H=1, L=15$, $\alpha_{0}=\pi / 3, a=1, b=0.05$ and $c=0.05$. (a) Component $\mathrm{T}_{R, 33}$. (b) Component $\mathrm{T}_{R, 11}=$ $\mathrm{T}_{R, 22}$. (c) Component $\mathrm{T}_{R, 12}$. (d) Component $\mathrm{T}_{R, 21}$.

Always with reference to the case considered in Figure 10, Figure 11 the stress distributions along the four sides of the cross section are plotted (for a better view of diagrams in Figure 11(b), the component $\mathrm{T}_{R, 12}$ was multiplied by a factor of 200 with respect to normal components $\mathrm{T}_{R, 11}=\mathrm{T}_{R, 22}$ ). Although these stress distributions are not exactly null as prescribed by boundary conditions 20 , their numerical values are however very small and therefore conditions 20 may be considered approximately fulfilled.

${ }_{406}$ A further check can be performed by calculating the normal force and verifying that it is close to zero. In the cross section $Z=0$, the normal force is 408 given by

$$
N=\int \mathrm{T}_{R, 33} d A=\int_{-\frac{B}{2}}^{\frac{B}{2}} \int_{-\frac{H}{2}}^{\frac{H}{2}} \mathrm{~S}_{Z} d Y d X,
$$



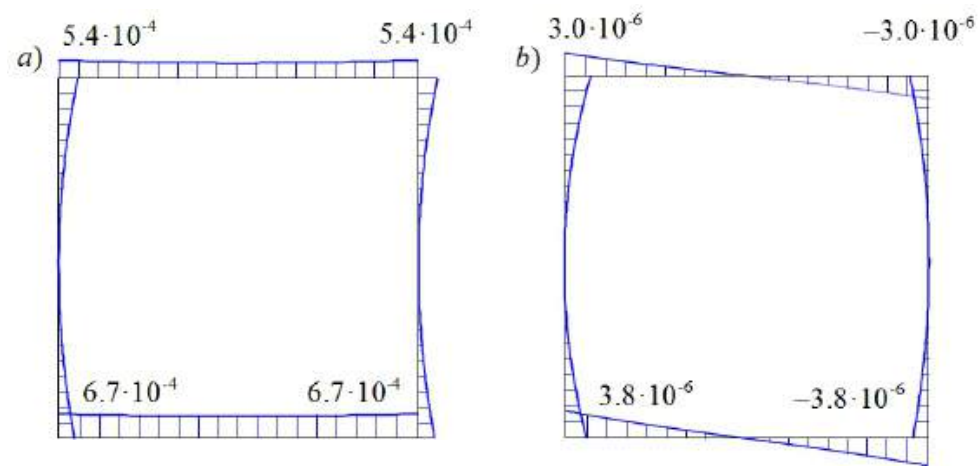

Figure 11: Plot of the stress distributions evaluated along the edges of the cross section $Z=0$. Adopted parameters: $B=1, H=1, L=15, \alpha_{0}=\pi / 3, a=1, b=0.05$ and $c=0.05$. (a) Component $\mathrm{T}_{R, 11}=\mathrm{T}_{R, 22}$. (b) Component $\mathrm{T}_{R, 12}=-\mathrm{T}_{R, 21}$.

where $\mathrm{S}_{Z}=2 \lambda_{Z}\left(a+2 b \lambda^{2}+c \lambda^{4}\right)-\frac{d}{\lambda_{Z}} 7$ Even the normal force $N$ is dimensionless.

In Figure $12(\mathrm{a}), N$ is plotted for the reference beam by varying both $\alpha_{0}$ and $L$. As can be seen from Figure $12(\mathrm{a}) \quad N$ is small and it becomes even smaller when $r$ grows.
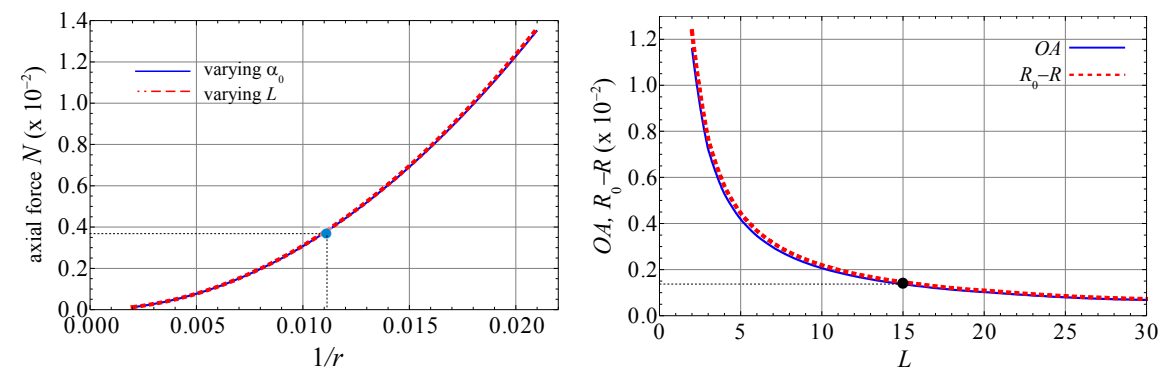

(a) Normal force $N$ versus the curvature (b) Geometrical quantities $O A$ and $\left(R_{0}-R\right)$ $1 / r$. versus the length of the beam $L$.

Figure 12: Plot of the axial force $N$ varying $1 / r$ and geometrical quantities $O A$ and $\left(R_{0}-R\right)$ versus $L$. The dotted line represents the reference beam.

${ }^{7}$ Using $[9]$, it can be promptly verified that in the absence of deformation $\left(\lambda=\lambda_{Z}=1\right)$ is $\mathrm{S}_{Z}=N=0$. 
In closing this Section, we make an interesting observation. The geometrical Figure 2 is modified as depicted by Figure 13.
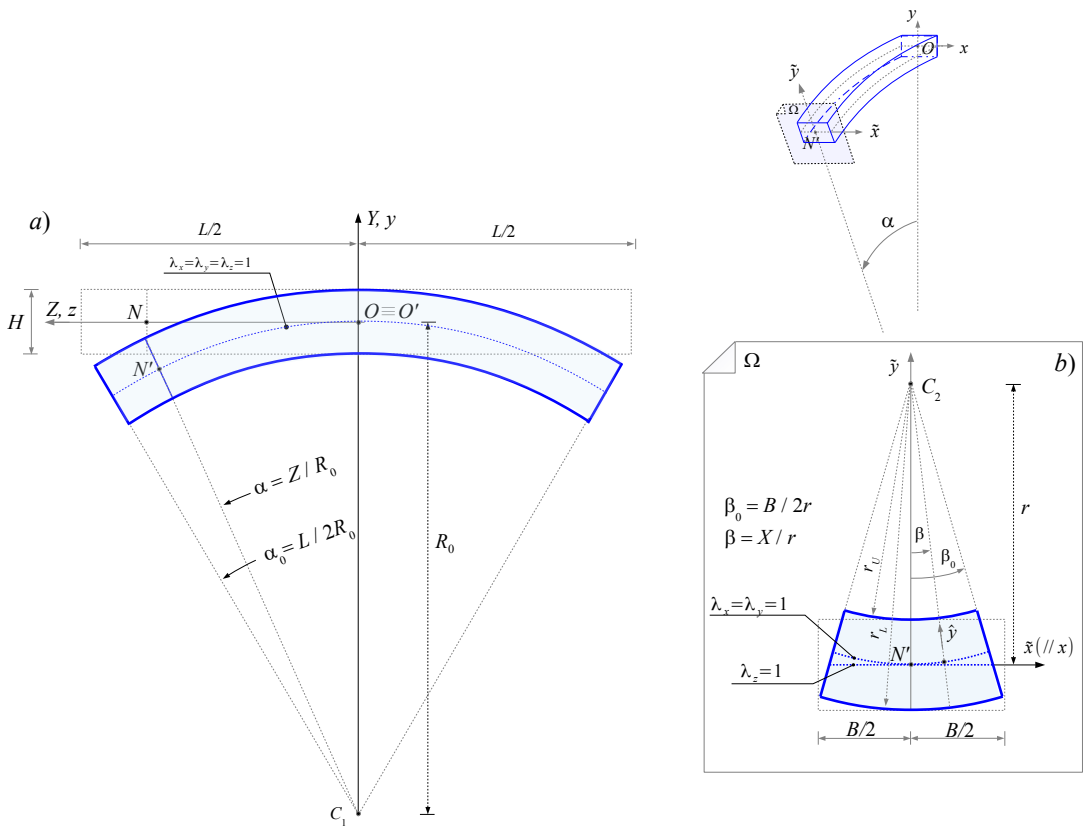

Figure 13: Deformation of a slender beam. a) Longitudinal deformation in the vertical $Y Z$ plane. b) Transversal deformation in the generic cross section $\Omega$. 
For slender beams, the displacement field (4) transforms into

$$
\left\{\begin{array}{l}
u=-X+r e^{-\frac{Y}{r}} \sin \frac{X}{r} \\
v=-Y-R_{0}+\left[R_{0}+r\left(1-e^{-\frac{Y}{r}} \cos \frac{X}{r}\right)\right] \cos \frac{Z}{R_{0}} \\
w=-Z+\left[R_{0}+r\left(1-e^{-\frac{Y}{r}} \cos \frac{X}{r}\right)\right] \sin \frac{Z}{R_{0}}
\end{array}\right.
$$

${ }_{424}$ In this system there is a single unknown kinematic parameter: the transversal radius $r$. It can be determined using the following relation:

$$
(b+c) r-(a+3 b+2 c) R_{0}=0,
$$

426

derived from (5). The expression (23) governs the equilibrium of the points belonging to the longitudinal basic line: $X=0, Y=0$ and $Z=Z 8$

From 22 , the following expressions for the principal stretches are obtained:

$$
\left\{\begin{array}{l}
\lambda_{X}=\lambda_{Y}=e^{-\frac{Y}{r}} \\
\lambda_{Z}=1+\frac{r}{R_{0}}\left(1-e^{-\frac{Y}{r}} \cos \frac{X}{r}\right)
\end{array} .\right.
$$

Formula (11) for the deformation gradient $\mathbf{F}$, eqn $(12)$ for the rotation tensor ${ }_{430} \mathbf{R}$, eqn 13 for the stretch tensor $\mathbf{U}$ and eqn 15 for the Piola-Kirchhoff stress tensor $\mathbf{T}_{R}$ is still valid provided that for the stretches the expressions $(24)$ are used.

In the deformed configuration, stretches and stresses are expressed in terms of Eulerian coordinates $(x, y, z)$. Using eqns $(2)$ and $(22)$ the Eulerian coordi-

\footnotetext{
${ }^{8}$ Since $O A$ and $\left(R_{0}-R\right)$ are assumed to vanish, 6 and 7 no longer serve. Indeed, in the case of slender beams, these formulae can be regarded as fulfilled because the transversal radius $r$ is usually much larger than the dimensions $B$ and $H$ of the cross section. In this way, two conditions, characterized by a certain degree of approximation, are eliminated from the problem formulation.
} 
nates of a generic point of the beam are

$$
\left\{\begin{array}{l}
x=X+u=r e^{-\frac{Y}{r}} \sin \frac{X}{r} \\
y=Y+v=-R_{0}+\left[R_{0}+r\left(1-e^{-\frac{Y}{r}} \cos \frac{X}{r}\right)\right] \cos \frac{Z}{R_{0}} \\
z=Z+w=\left[R_{0}+r\left(1-e^{-\frac{Y}{r}} \cos \frac{X}{r}\right)\right] \sin \frac{Z}{R_{0}}
\end{array}\right.
$$

436 The inversion of this coupled system provides the following expressions 99

$$
\left\{\begin{array}{l}
X=r \arctan \left[\frac{x}{\left.R_{0}+r-\frac{y+R_{0}}{\cos \left(\arctan \frac{z}{y+R_{0}}\right)}\right]}\right] \\
Y=-r \ln \left\{\frac{R_{0}+r-\frac{y+R_{0}}{\cos \left[\arctan \left(\frac{z}{y+R_{0}}\right)\right]}}{r \cos \left\{\arctan \left(\frac{x}{R_{0}+r-\frac{y+R_{0}}{\cos \left(\arctan \frac{z}{y+R_{0}}\right)}}\right)\right\}}\right\} \\
Z=R_{0} \arctan \left(\frac{z}{y+R_{0}}\right)
\end{array}\right.
$$

that formally allow the transition from Lagrangian coordinates to Eulerian co-

Due to the deformation, the longitudinal basic line transforms in a arc of circumference. The generic point $N^{\prime}$ of this curve has the following spatial coordinates:

$$
N^{\prime}=\left(0,-R_{0}(1-\cos \alpha), R_{0} \sin \alpha\right),
$$

${ }_{442}$ where $\alpha=\arctan \left(\frac{z}{y+R_{0}}\right)$. The point $N^{\prime}$ is the origin of the reference system $(\tilde{x}, \tilde{y})$ of the cross section $\Omega(c f$. Figure $13(\mathrm{~b}))$.

\footnotetext{
${ }^{9}$ From 25$]_{3}$ the quantity in square brackets is attained and then replaced into 25$]_{2}$, obtaining 26] 3 . Similarly, from $251, r e^{-\frac{Y}{r}}$ is evaluated and then substituted into $252_{2}$, obtaining $[26]_{1}$. Expression $[26]_{2}$ is evaluated directly from $[25]_{2}$ using $[26]_{1}$ and 26$]_{3}$.
} 
Substituting (26) into (24), the principal stretches $\lambda_{x}, \lambda_{y}$ and $\lambda_{z}$ are obtained

$$
\left\{\begin{array}{l}
\lambda_{x}=\lambda_{y}=\frac{R_{0}+r-\frac{y+R_{0}}{\cos \left[\arctan \left(\frac{z}{y+R_{0}}\right)\right]}}{r \cos \left(\operatorname { a r c t a n } \left[\frac{x}{\left.\left.R_{0}+r-\frac{y+R_{0}}{\cos \left(\arctan \frac{z}{y+R_{0}}\right)}\right]\right)} .\right.\right.} . \\
\lambda_{z}=1+\frac{1}{R_{0}}\left\{\frac{y+R_{0}}{\cos \left[\arctan \left(\frac{z}{y+R_{0}}\right)\right]}-R_{0}\right\}
\end{array}\right.
$$

These Eulerian expressions are more complicated than the corresponding La-

446

rial variable $Y$, now, in the deformed configuration, the corresponding stretches $\lambda_{x}=\lambda_{y}$ depend on all three spatial variables $x, y$ and $z$. Note also that $\lambda_{z}$ loses the dependence on spatial variable $x$.

As shown in Figure 13(b), for the generic cross section $\Omega$, the stretch $\lambda_{z}$ is unitary along the horizontal straight line passing through $N^{\prime}$. This line $(\tilde{y}=0)$ can be considered as the neutral axis for the deformation. Moreover, $\lambda_{z}$ has the same diagram along all vertical straight lines of cross section $\Omega$, that is ${ }_{454}$ by varying the abscissa $\tilde{x}$ and holding the $\alpha$ angle fixed. The shape of such a vertical diagram is linear in $\tilde{y}$

$$
\lambda_{z}=1+\frac{\tilde{y}}{R_{0}} .
$$

This simple and compact formula is obtained by introducing the variable $\tilde{y}=$ $\frac{y+R_{0}}{\cos \alpha}-R_{0}$ into $(28)_{2}$.

458

It is important to note that the linear laws obtained for the stretch $\lambda_{z}$ and for its neutral axis are direct consequences of the kinematic model adopted. In fact, given the hypothesis of the planarity preservation for cross sections, all cross sections of the beam deform maintaining own plane and rotating around the neutral axis. The rotation is finite. This kind of deformation leads directly to expression 29 for the longitudinal stretch $\lambda_{z}$ in the deformed configuration.

The stretches $\lambda_{x}=\lambda_{y}$ are unitary along the circle arc with radius $r$, concavity upwards and passing through the point $N^{\prime}$ (cf. Figure 13(b)). Even for 
29. In fact, using $\tilde{y}$ and introducing the new variable $\hat{y}=\frac{\tilde{y}-r(1-\cos \beta)}{\cos \beta}$, where ${ }_{468} \beta=\arctan \left(\frac{x}{r-\tilde{y}}\right), 28{ }_{1}$ transforms into

$$
\lambda_{x}=\lambda_{y}=1-\frac{\hat{y}}{r} .
$$

As shown by this equation, the transversal stretches $\lambda_{x}=\lambda_{y}$ are linear along the $\hat{y}$ direction. In addition, their diagrams are the same as themselves along the curved cross section profile. That is, they are the same for each $\beta$ angle.

To apply formulae $(29)$ and $(30)$ the coordinates $\tilde{y}$ and $\hat{y}$ for each point of the deformed beam can be evaluated by using $(25)$, whereas the radius $R_{0}$ is 474 given by the boundary conditions and the radius $r$ can be computed directly from $(23)$.

476 Figure 14 shows a comparison between Lagrangian and Eulerian stretches evaluated for the middle cross section of a slender beam. To emphasize the
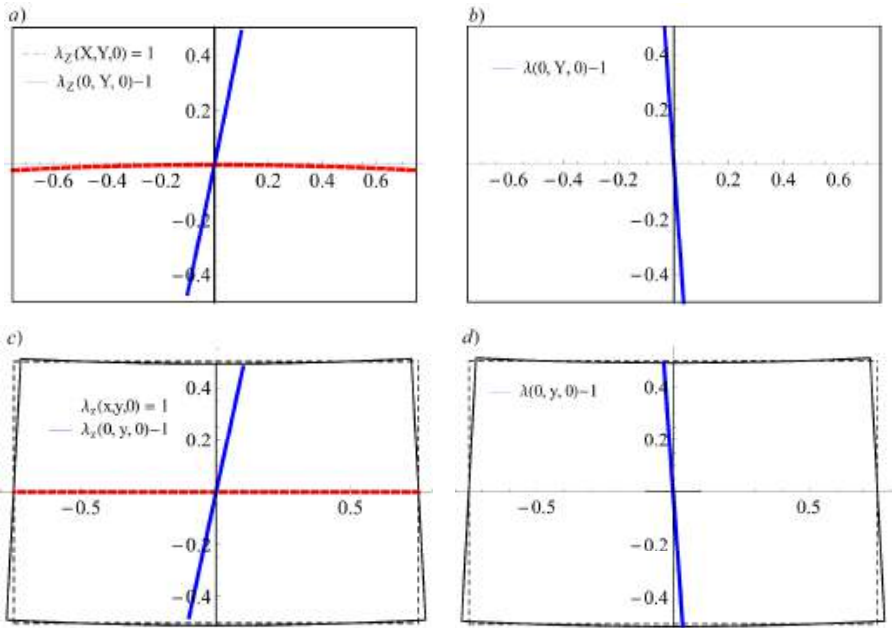

Figure 14: Diagrams of the stretches minus one along the vertical lines $x=X=0$ of the middle cross section of a slender beam. Adopted parameters: $B=3 / 2, H=1, L=15, \alpha_{0}=\pi / 2$, $a=b=c=1$. Longitudinal and transversal stretches in the reference configuration. (a) $\lambda_{Z}-1$. (b) $\lambda_{X}-1=\lambda_{Y}-1$. Longitudinal and transversal stretches in the deformed configuration. (c) $\lambda_{z}-1$. (d) $\lambda_{x}-1=\lambda_{y}-1$. 
478 To distinguish between dilations and contractions, in this figure the functions ${ }_{480}(\lambda-1)$ are plotted. The cross section is thus divided into two parts by the line $\lambda=1$. In the part where $(\lambda-1)$ is positive there is dilatation and in the other ${ }_{482}$ part, where $(\lambda-1)$ is negative, there is contraction. For the vertical line $X=0$ of the middle cross section of the undeformed beam, in Figure 14(a) and 14(b) the diagrams of $\left(\lambda_{Z}-1\right)$ and $\left(\lambda_{X}-1\right)$ are drawn. These functions were obtained by using (24) and they show a quasi-rectilinear shape, since the exponent $(-Y / r)$ of the exponential function is a small quantity. In Figure 14 (a), with a dotted line, the curve $\lambda_{Z}=1$ is displayed. This curve, which shows the concavity facing downward, after the inflexion of the beam will become perfectly straight ( $c f$. Figure 14(c)). Figure 14(c) and 14(d) show the functions $\left(\lambda_{z}-1\right)$ and $\left(\lambda_{x}-1\right)$ in the deformed cross section. The Eulerian stretches 28 for $x=z=0$, or equivalently 29 and 30 for $\alpha=\beta=0$, provide the following liner expressions:

$$
\left\{\begin{array}{l}
\lambda_{x}=\lambda_{y}=1-\frac{y}{r} \\
\lambda_{z}=1+\frac{y}{R_{0}}
\end{array},\right.
$$

which were used to plot Figures $14(\mathrm{c})$ and $14(\mathrm{~d})$.

Considering the longitudinal profile of the inflexed beam, in Figure 15 some diagrams of $\left(\lambda_{z}-1\right)$ are shown for discrete values of the variable $z$. The stretch $\lambda_{z}$ is evaluated by $(28)_{2}$. The parameters adopted are those of the beam of 496 Figure 14. As can be noted in Figure 15, for all cross sections the same linear diagram is obtained. At the upper fibre, $\lambda_{z, \max }=1.1029$, while at the lower fibre $\lambda_{z, \min }=0.8934$. Obviously, this result is immediately apparent from (29) which predicts the same diagram for each $\alpha$ angle.

In the sequel, using the results obtained so far, some geometrical quantities of the beam in the deformed configuration are computed. The radii of curvature of the lower $\left(r_{L}\right)$ and upper $\left(r_{U}\right)$ fibres of the cross section after bending $(c f$. Figure 13) turn out to be $\left(r_{L}>r_{U}\right)$

$$
r_{L}=r+\int_{-\frac{H}{2}}^{0} \lambda_{Y}(\hat{Y}) d \hat{Y}=r e^{\frac{H}{2 r}}, \quad r_{U}=r-\int_{0}^{\frac{H}{2}} \lambda_{Y}(\hat{Y}) d \hat{Y}=r e^{-\frac{H}{2 r}}
$$




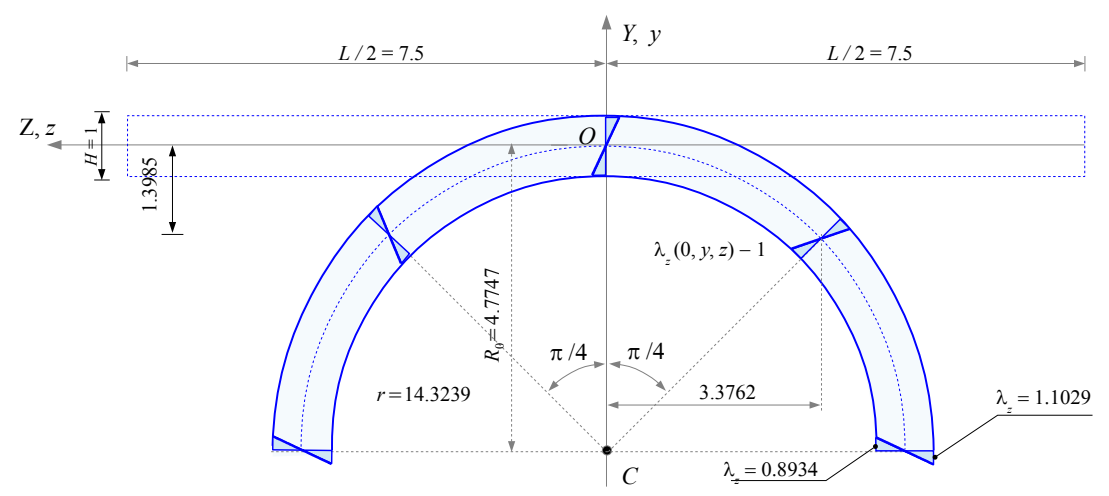

Figure 15: Longitudinal distribution of diagrams $\left(\lambda_{z}-1\right)$ in the deformed configuration.

The difference between these two radii is the radial thickness

$$
H^{\prime}=2 r \sinh \left(\frac{H}{2 r}\right)
$$

which is constant. Knowing the external radii $r_{L}$ and $r_{U}$ it is possible to evaluate the area of the deformed cross section $A^{\prime}$ as

$$
A^{\prime}=\int_{-\beta_{0}}^{\beta_{0}} d \vartheta \int_{r_{U}}^{r_{L}} \rho d \rho=2 r^{2} \beta_{0} \sinh \left(\frac{H}{r}\right),
$$

where $(\rho, \vartheta)$ is a polar coordinate system with pole at the point $C_{2}$ of Figure 13 (b). The distance between the centroid $G$ of the deformed cross section and the pole $C_{2}$ is

$$
C_{2} G=\frac{1}{A^{\prime}} \int_{-\beta_{0}}^{\beta_{0}} \cos \vartheta d \vartheta \int_{r_{U}}^{r_{L}} \rho^{2} d \rho=\frac{2}{3} r \frac{\sin \beta_{0}}{\beta_{0}} \frac{\sinh \left(\frac{3 H}{2 r}\right)}{\sinh \left(\frac{H}{r}\right)} .
$$

According to the second Pappus-Guldinus theorem, the volume of the inflexed beam $V^{\prime}$ can be calculated as the volume of revolution generated by the rotation of $A^{\prime}$ around $C_{1}$. Thus, with (34) and (35), the following expression is 
obtained 10

$$
\begin{aligned}
V^{\prime} & =2 \alpha_{0}\left(R_{0}+r-C_{2} G\right) A^{\prime} \\
& =\frac{4}{3} \alpha_{0} r^{2}\left[3 \beta_{0}\left(R_{0}+r\right)-2 r \frac{\sinh \left(\frac{3 H}{2 r}\right)}{\sinh \left(\frac{H}{r}\right)} \sin \beta_{0}\right] \sinh \left(\frac{H}{r}\right) .
\end{aligned}
$$

Let us now consider the stresses. Lagrangian stresses are expressed by the Piola-Kirchhoff stress tensor $\mathbf{T}_{R}(c f$. eqn $(15)$ ). The stress measure coherently employed in the spatial configuration is instead that of Cauchy. The Cauchy stress tensor $\mathbf{T}$ is obtained from the Piola-Kirchhoff stress tensor $\mathbf{T}_{R}$ through the well-known transformation

$$
\mathbf{T}_{R}=\mathbf{T} \mathbf{F}^{\star}
$$

Using 11], 12), 13, , 15 and (37), the following components of the Cauchy stress tensor are computed $\left(\mathrm{S}=\mathrm{S}_{X}=\mathrm{S}_{Y}, \lambda=\lambda_{X}=\lambda_{Y}\right)$ :

$$
\lambda^{2} \lambda_{Z}[\mathbf{T}]=\left[\begin{array}{ccc}
\mathrm{S} \lambda & 0 & 0 \\
0 & \mathrm{~S} \lambda \cos ^{2} \alpha+\mathrm{S} Z \lambda_{Z} \sin ^{2} \alpha & \left(\mathrm{S} \lambda-\mathrm{S}_{Z} \lambda_{Z}\right) \sin \alpha \cos \alpha \\
0 & \left(\mathrm{~S} \lambda-\mathrm{S}_{Z} \lambda_{Z}\right) \sin \alpha \cos \alpha & \mathrm{S} \lambda \sin ^{2} \alpha+\mathrm{S}_{Z} \lambda_{Z} \cos ^{2} \alpha
\end{array}\right],
$$

being $\mathbf{F}^{\star}=\left(\lambda^{2} \lambda_{Z}\right) \mathbf{R} \mathbf{U}^{-1}$ and $\left(\lambda^{2} \lambda_{Z}\right) \mathbf{T}=\mathbf{R} \mathbf{S} \mathbf{U} \mathbf{R}^{T}$. The tensor $\mathbf{T}$ is sym522 metric. The matrix (38) can be rewritten in diagonal form by evaluating its eigenvalues. The resolution of the characteristic polynomial allows the determination of the principal Cauchy stresses

$$
[\mathbf{T}]=\left[\begin{array}{ccc}
\frac{\mathrm{S}}{\lambda \lambda_{Z}} & 0 & 0 \\
0 & \frac{\mathrm{S}}{\lambda \lambda_{Z}} & 0 \\
0 & 0 & \frac{S_{Z}}{\lambda^{2}}
\end{array}\right]
$$

where

$$
\begin{aligned}
& \mathrm{T}_{1}=\mathrm{T}_{2}=\frac{\mathrm{S}}{\lambda \lambda_{Z}}=\frac{2}{\lambda_{Z}}\left[\omega_{1}+\left(\lambda^{2}+\lambda_{Z}^{2}\right) \omega_{2}+\lambda^{2} \lambda_{Z}^{2} \omega_{3}\right], \\
& \mathrm{T}_{3}=\frac{\mathrm{S}_{Z}}{\lambda^{2}}=\frac{2 \lambda_{Z}}{\lambda^{2}}\left(\omega_{1}+2 \lambda^{2} \omega_{2}+\lambda^{4} \omega_{3}\right) .
\end{aligned}
$$

\footnotetext{
${ }^{10}$ It can be see that, by taking $B=2 \beta_{0} r$, 34 reduces to $B H$ as $r \rightarrow \infty$. Similarly, by taking $L=2 \alpha_{0} R_{0}$, 36 becomes $V^{\prime}=B H L$ as $\left(R_{0}, r\right) \rightarrow \infty$.
} 
The principal directions of stress are the eigenvectors associated with these eigenvalues. The principal direction corresponding to the eigenvalue $T_{3}$ is the unit vector orthogonal to the plane $\Omega$ (cf. Figure 13 ) with components (0, $-\sin \alpha, \cos \alpha$ ). The others two eigenvectors are any two unit vectors orthogonal to each other and belonging to the plane $\Omega$. Substituting (9) and 19 in 40 , the principal Cauchy stresses for a compressible Mooney-Rivlin material are obtained

$$
\begin{aligned}
& \mathrm{T}_{1}=\mathrm{T}_{2}=\frac{2}{\lambda^{2} \lambda_{Z}}\left\{\left(\lambda^{2}-1\right) a+\left[\left(\lambda^{2}+\lambda_{Z}^{2}\right) \lambda^{2}-2\right] b+\left(\lambda^{4} \lambda_{Z}^{2}-1\right) c\right\}, \\
& \mathrm{T}_{3}=\frac{2}{\lambda^{2} \lambda_{Z}}\left[\left(\lambda_{Z}^{2}-1\right) a+2\left(\lambda^{2} \lambda_{Z}^{2}-1\right) b+\left(\lambda^{4} \lambda_{Z}^{2}-1\right) c\right] .
\end{aligned}
$$

Note that in the absence of deformation these stresses vanish. To represent stresses in the deformed configuration is necessary to use the expressions (28) for the stretches.

Figure 16 shows a comparison between Piola-Kirchhoff and Cauchy stresses evaluated, locally in every point belonging to the middle cross section of the beam, in the undeformed and deformed configuration, respectively. PiolaKirchhoff stresses are calculated using (15) with $\alpha=0$ and Cauchy stresses by (38) still for $\alpha=0$. As can be seen from this figure, the longitudinal stress components $\mathrm{T}_{R, 33}$ and $\mathrm{T}_{33}$ are considerably greater than the in-plane components, which are close to zero. Furthermore, for the longitudinal stress components, the following can be observed. In the upper half of the cross section there are 54 tensile stresses and in the lower one there are compression stresses. In absolute value, maximum levels of stress are reached at the upper and lower edges ( $c f$. ${ }_{546}$ Figures $16(\mathrm{a})$ and $\left.16(\mathrm{c})\right)$. Figure 17 continues to provide information on the stresses shown in Figure 16, but it is now organized as Figure 14, previously 54 plotted for stretches. Figure 17 shows the diagrams of the stress along the vertical lines $X=Z=0$ and $x=z=0$. To compute Piola-Kirchhoff stresses, which are principal, eqn 15ith $\alpha=\beta=0$ has been used. The Cauchy principal stresses are calculated by using 41. Though the corresponding stretch diagrams of Figures 14(c) and 14(d) are linear, the diagrams of Figures 17(c) and 17(d) are nonlinear. In Figure 17(c), with a dashed line, the neutral line for 

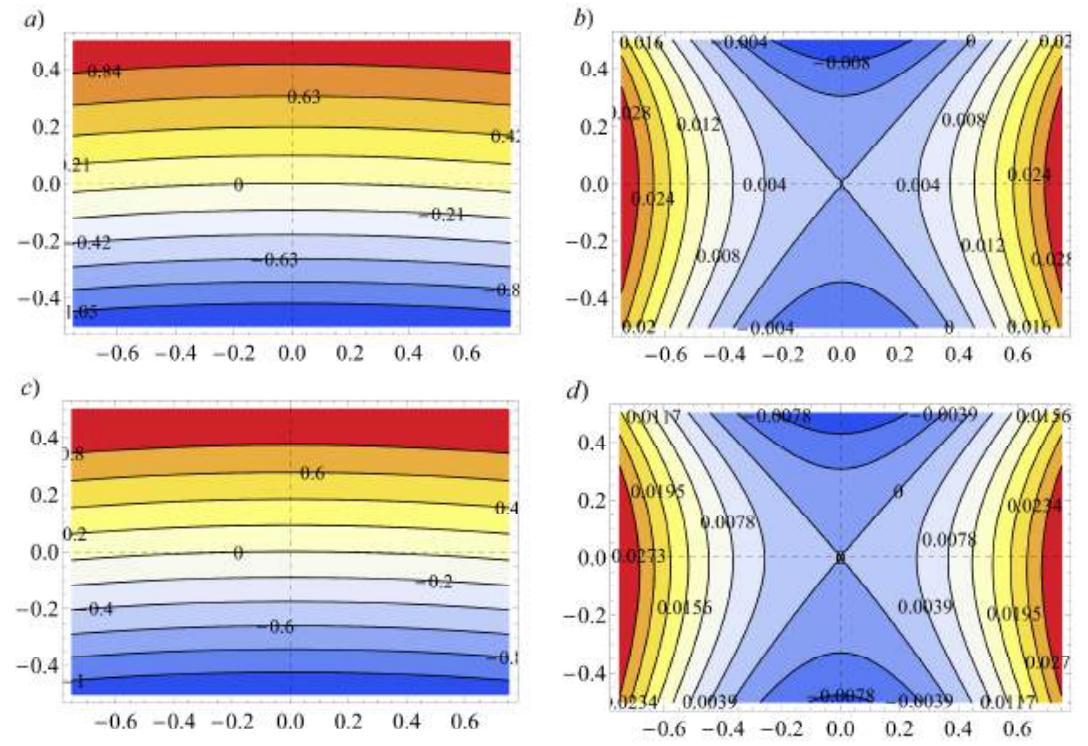

Figure 16: Plot of the components of the stress evaluated locally in every point belonging to the middle cross section $Z=z=0$ of a slender beam. Adopted parameters: $B=3 / 2, H=$ 1, $L=15, \alpha_{0}=\pi / 2, a=b=c=1$. Piola-Kirchhoff stresses. (a) Component $\mathrm{T}_{R, 33}$. (b) Components $\mathrm{T}_{R, 11}=\mathrm{T}_{R, 22}$. Cauchy stresses. (c) Component $\mathrm{T}_{33}$. (d) Components $\mathrm{T}_{11}=\mathrm{T}_{22}$.

which $\mathrm{T}_{3}=0$ is also pointed out. Although the two lines are near, the neutral curve for the stresses does not coincide with the neutral axis for the deformation $\lambda_{z}=0$.

The longitudinal diagrams of the Cauchy principal stress $\mathrm{T}_{3}$ are shown in Figure 18. As in the case of the stretches illustrated by Figure 15, the stress diagrams are all equal along the curved profile of the beam. At the upper fibre, $\mathrm{T}_{3}=1.05606$ (tensile), while at the lower fibre $\mathrm{T}_{3}=-1.19428$ (compression).

Knowing the stress distributions, the normal force $n$ and the bending moment $m_{x}$ can be evaluated. Using the polar coordinate system $(\rho, \beta)$ of Figure 13 (b), for the generic deformed cross section $\Omega$ the following expressions are 564 derived:

$$
n=\int_{-\beta_{0}}^{\beta_{0}} \int_{\rho_{\min }}^{\rho_{\max }} \rho \mathrm{T}_{3}(\rho, \beta) d \rho d \beta, \quad m_{x}=\int_{-\beta_{0}}^{\beta_{0}} \int_{\rho_{\min }}^{\rho_{\max }} \rho \mathrm{T}_{3}(\rho, \beta) \tilde{y} d \rho d \beta,
$$



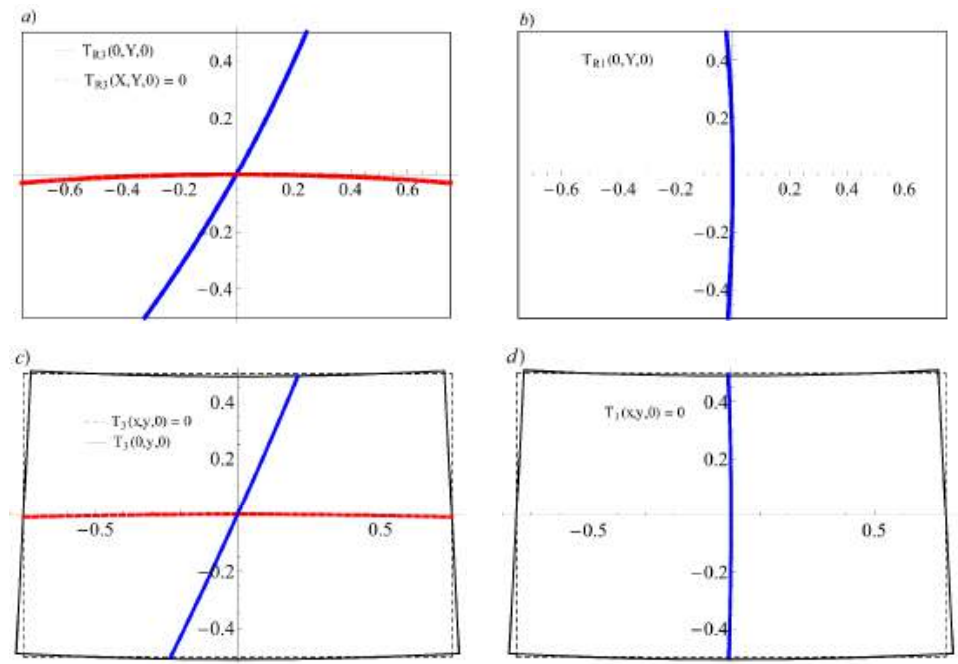

Figure 17: Diagrams of principal stresses along the vertical lines $x=X=0$ of the middle cross section of a slender beam. Adopted parameters: $B=3 / 2, H=1, L=15, \alpha_{0}=\pi / 2$, $a=b=c=1$. Piola-Kirchhoff stresses. (a) Component $\mathrm{T}_{R, 3}$. (b) Components $\mathrm{T}_{R, 1}=\mathrm{T}_{R, 2}$. Cauchy stresses. (c) Component $\mathrm{T}_{3}$. (d) Components $\mathrm{T}_{1}=\mathrm{T}_{2}$.

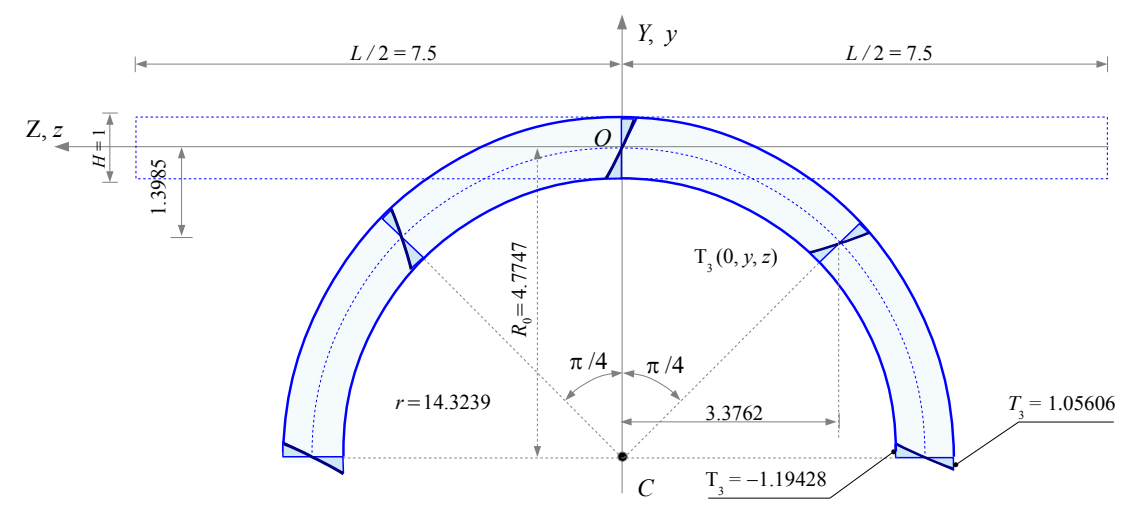

Figure 18: Longitudinal distribution of diagrams of Cauchy principal stress $\mathrm{T}_{3}$ in the deformed configuration.

where $\tilde{x}=\rho \sin \beta, \tilde{y}=r-\rho \cos \beta, \rho_{\min }=r_{U}=r e^{-\frac{H}{2 r}}, \rho_{\max }=r_{L}=r e^{\frac{H}{2 r}}$.

The modulus of the above internal actions does not depend on the cross section 
$\Omega$.

In terms of polar coordinates $(\rho, \beta)$, the stretches 28 , for the middle cross section $z=0$, assume the following form:

$$
\left\{\begin{array}{l}
\lambda=\lambda_{x}=\lambda_{y}=\frac{\rho}{r} \\
\lambda_{z}=1+\frac{r-\rho \cos \beta}{R_{0}}
\end{array} .\right.
$$

Substituting $(41)_{2}$ and 43 into $421_{1}$, the normal force $n$ is obtained as the sum of four integrals

$$
\begin{aligned}
& 2 a \int_{-\beta_{0}}^{\beta_{0}} \int_{\rho_{\min }}^{\rho_{\max }} \frac{\lambda_{z}}{\lambda^{2}} \rho d \rho d \beta=2 a\left(1+\frac{r}{R_{0}}\right) B H-8 a \frac{r^{3}}{R_{0}} \sinh \left(\frac{H}{2 r}\right) \sin \left(\frac{B}{2 r}\right), \\
& 4 b \int_{-\beta_{0}}^{\beta_{0}} \int_{\rho_{\min }}^{\rho_{\max }} \lambda_{z} \rho d \rho d \beta=4 b\left(1+\frac{r}{R_{0}}\right) r B \sinh \left(\frac{H}{r}\right)-\frac{16}{3} b \frac{r^{3}}{R_{0}} \sinh \left(\frac{3 H}{2 r}\right) \sin \left(\frac{B}{2 r}\right), \\
& 2 c \int_{-\beta_{0}}^{\beta_{0}} \int_{\rho_{\min }}^{\rho_{\max }} \lambda^{2} \lambda_{z} \rho d \rho d \beta=c\left(1+\frac{r}{R_{0}}\right) r B \sinh \left(\frac{2 H}{r}\right)-\frac{8 c}{5} \frac{r^{3}}{R_{0}} \sinh \left(\frac{5 H}{2 r}\right) \sin \left(\frac{B}{2 r}\right), \\
& -(2 a+4 b+2 c) \int_{-\beta_{0}}^{\beta_{0}} \int_{\rho_{\min }}^{\rho_{\max }} \frac{\rho}{\lambda^{2} \lambda_{z}} d \rho d \beta=-(2 a+4 b+2 c) \frac{r R_{0}}{\left(r+R_{0}\right)}\left\{\frac{B H}{r}\right. \\
& \left.-B \ln \left[\frac{r+R_{0}-r e^{\frac{H}{2 r}}}{r+R_{0}-r e^{-\frac{H}{2 r}}}\right]+\frac{\left(r+R_{0}\right) B^{3} \sinh \left(\frac{H}{2 r}\right)}{12 r\left[2 r\left(r+R_{0}\right) \cosh \left(\frac{H}{2 r}\right)-\left(2 r^{2}+2 r R_{0}+R_{0}^{2}\right)\right]}\right\} .
\end{aligned}
$$

Similarly, the bending moment $m_{x}$ is provided by the sum of the following four integrals:

$$
\begin{gathered}
2 a \int_{-\beta_{0}}^{\beta_{0}} \int_{\rho_{\min }}^{\rho_{\max }} \frac{\lambda_{z}}{\lambda^{2}}(r-\rho \cos \beta) \rho d \rho d \beta=2 a\left(1+\frac{r}{R_{0}}\right) r B H \\
\quad-8 a\left(1+\frac{2 r}{R_{0}}\right) r^{3} \sinh \left(\frac{H}{2 r}\right) \sin \left(\frac{B}{2 r}\right)+a \frac{r^{4}}{R_{0}} \sinh \left(\frac{H}{r}\right)\left[\sin \left(\frac{B}{r}\right)+\frac{B}{r}\right], \\
4 b \int_{-\beta_{0}}^{\beta_{0}} \int_{\rho_{\min }}^{\rho_{\max }} \lambda_{z}(r-\rho \cos \beta) \rho d \rho d \beta=4 b\left(1+\frac{r}{R_{0}}\right) r^{2} B \sinh \left(\frac{H}{r}\right) \\
\quad-\frac{16}{3} b\left(1+\frac{2 r}{R_{0}}\right) r^{3} \sinh \left(\frac{3 H}{2 r}\right) \sin \left(\frac{B}{2 r}\right)+b \frac{r^{4}}{R_{0}} \sinh \left(\frac{2 H}{r}\right)\left[\sin \left(\frac{B}{r}\right)+\frac{B}{r}\right], \\
2 c \int_{-\beta_{0}}^{\beta_{0}} \int_{\rho_{\min }}^{\rho_{\max }} \lambda^{2} \lambda_{z}(r-\rho \cos \beta) \rho d \rho d \beta=c\left(1+\frac{r}{R_{0}}\right) r^{2} B \sinh \left(\frac{2 H}{r}\right) \\
\quad-\frac{8}{5} c\left(1+\frac{2 r}{R_{0}}\right) r^{3} \sinh \left(\frac{5 H}{2 r}\right) \sin \left(\frac{B}{2 r}\right)+\frac{1}{3} c \frac{r^{4}}{R_{0}} \sinh \left(\frac{3 H}{r}\right)\left[\sin \left(\frac{B}{r}\right)+\frac{B}{r}\right], \\
-(2 a+4 b+2 c) \int_{-\beta_{0}}^{\beta_{0}} \int_{\rho_{\min }}^{\rho_{\max }} \frac{1}{\lambda^{2} \lambda_{z}}(r-\rho \cos \beta) \rho d \rho d \beta=-(2 a+4 b+2 c)\left\{\frac{r R_{0} B}{\left(r+R_{0}\right)}[H\right. \\
\left.\left.\quad-R_{0} \ln \left(\frac{r+R_{0}-r e^{-\frac{H}{2 r}}}{r+R_{0}-r e^{\frac{H}{2 r}}}\right)\right]-\frac{R_{0}^{2} B^{3}\left(e^{\frac{H}{r}}-1\right)}{24\left[\left(e^{\frac{H}{2 r}}-1\right) r-R_{0}\right]\left[\left(e^{\frac{H}{2 r}}-1\right) r+R_{0} e^{\frac{H}{2 r}}\right]}\right\} .
\end{gathered}
$$

To simplify the calculus of integrals $(44)_{4}$ and $(45)_{4}$, after the first integration in the variable $\rho$, the integrand functions have been developed in power series around $\beta=0$, taking into account the powers up to $\beta^{2}$. 
As the corresponding Lagrangian normal force $N$ (cf. Figure 12(a)), the

2.4. Linearization

590 smaller for $L$ increasing.

Expression $422_{2}$, with integrals 45 , can be thought of as the momentcurvature relationship for nonlinear beams, $m_{x}=\hat{m}_{x}\left(R_{0}^{-1}\right)$. In addition, since $\alpha_{0}=L / 2 R_{0}$, the $(42)_{2}$ can also be used to establish the correspondence between the moment $m_{x}$ and the angle $\alpha_{0}$. Pursuing this goal, the diagram plotted in Figure 19 has been obtained for the beam considered in Figures 14 18, In this last figure, the moment $m_{x}$ is assessed numerically by varying the angle $\alpha_{0}$. On the basis of this result, the boundary conditions at the two end faces can be set geometrically by means of the angle $\alpha_{0}$ or statically through the application of the corresponding moment $m_{x}$.

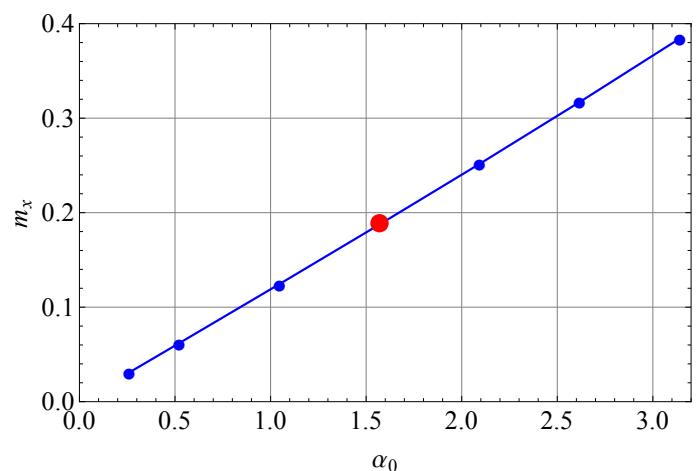

Figure 19: Plot of the bending moment $m_{x}$ versus the angle $\alpha_{0}$.

Enforcing the hypotheses of smallness of both the deformation and displacement fields, the theory exposed in Section 2.3 will be linearized, retrieving the classical results of the linear theory of inflexed beams. For this purpose, the main formulae will develop in power series as functions of the radii $r$ and $R_{0}$. These series will be truncated preserving the infinitesimals of order $\mathcal{O}\left(r^{-1}\right)$ and $\mathcal{O}\left(R_{0}^{-1}\right)$ as $r \rightarrow \infty$ or $R_{0} \rightarrow \infty$. Therefore, the displacement field 22 can be 
approximated as 11

$$
\begin{aligned}
u \simeq & -X+r\left[1-\frac{Y}{r}+\frac{Y^{2}}{2 r^{2}}+o\left(r^{-2}\right)\right]\left[\frac{X}{r}+o\left(r^{-2}\right)\right] \simeq-\frac{X Y}{r}+o\left(r^{-1}\right), \\
v \simeq & -Y-R_{0}+\left\{R_{0}+r-r\left[1-\frac{Y}{r}+\frac{Y^{2}}{2 r^{2}}+o\left(r^{-2}\right)\right]\left[1-\frac{X^{2}}{2 r^{2}}+o\left(r^{-3}\right)\right]\right\} \\
& {\left[1-\frac{Z^{2}}{2 R_{0}^{2}}+o\left(R_{0}^{-3}\right)\right] \simeq-\frac{Y^{2}}{2 r}+\frac{X^{2}}{2 r}-\frac{Z^{2}}{2 R_{0}}+o\left(r^{-1}\right)+o\left(R_{0}^{-1}\right), } \\
w \simeq & -Z+\left\{R_{0}+r-r\left[1-\frac{Y}{r}+\frac{Y^{2}}{2 r^{2}}+o\left(r^{-2}\right)\right]\left[1-\frac{X^{2}}{2 r^{2}}+o\left(r^{-3}\right)\right]\right\} \\
& {\left[\frac{Z}{R_{0}}+o\left(R_{0}^{-2}\right)\right] \simeq \frac{Y Z}{R_{0}}+o\left(r^{-1}\right)+o\left(R_{0}^{-1}\right), }
\end{aligned}
$$

having neglected the infinitesimal terms of higher order than $r^{-1}$ and $R_{0}^{-1}$. In

the linearized theory of inflexed beams, the following two well-known relationships hold:

$$
\frac{1}{R_{0}}=\frac{M_{X}}{E J_{X}}, \quad r=\frac{R_{0}}{\nu}
$$

where $M_{X}$ denotes the bending moment around the $X$ axis, $J_{X}$ the moment of inertia of the cross section with respect to the $X$ axis, $E$ the Young modulus and $\nu$ the Poisson ratio. The first equation represents the moment-curvature relationship and the second equation is derived from the ratio between the longitudinal and transversal radii. Introducing eqn (47) into 46, the infinitesimal displacement field is lastly derived ${ }^{12}$

$$
\left\{\begin{array}{l}
u \simeq-\frac{\nu M_{X}}{E J_{X}} X Y \\
v \simeq \frac{1}{2} \frac{M_{X}}{E J_{X}}\left[\nu\left(X^{2}-Y^{2}\right)-Z^{2}\right] \\
w \simeq \frac{M_{X}}{E J_{X}} Y Z
\end{array}\right.
$$

\footnotetext{
${ }^{11}$ The Landau symbols are used. In addition, in eqn 46 using the Taylor series expansions, the following approximations are employed:

$$
\begin{aligned}
& e^{-\frac{Y}{r}} \simeq 1-\frac{Y}{r}+\frac{Y^{2}}{2 r^{2}}+o\left(r^{-2}\right), \quad \sin \frac{X}{r} \simeq \frac{X}{r}+o\left(r^{-2}\right), \\
& \cos \frac{X}{r} \simeq 1-\frac{X^{2}}{2 r^{2}}+o\left(r^{-3}\right), \quad \sin \frac{Z}{R_{0}} \simeq \frac{Z}{R_{0}}+o\left(R_{0}^{-2}\right), \\
& \cos \frac{Z}{R_{0}} \simeq 1-\frac{Z^{2}}{2 R_{0}^{2}}+o\left(R_{0}^{-3}\right) .
\end{aligned}
$$
}

${ }^{12}$ In the sequel, the infinitesimal terms of higher order are omitted definitively. 
606

in the classic form which can be found in any textbook.

The linearization of the stretches 24 gives

$$
\left\{\begin{array}{l}
\lambda_{X}=\lambda_{Y} \simeq 1-\frac{Y}{r} \\
\lambda_{Z} \simeq 1+\frac{Y}{R_{0}}
\end{array},\right.
$$

608

and, subsequently, the deformation gradient $\mathbf{F}(11)$, the rotation tensor $\mathbf{R}(12$ and the stretch tensor $\mathbf{U}(13)$ transform into

$$
\begin{array}{ll}
{[\mathbf{F}] \simeq\left[\begin{array}{ccc}
1-\frac{Y}{r} & -\frac{X}{r} & 0 \\
\frac{X}{r} & 1-\frac{Y}{r} & -\frac{Z}{R_{0}} \\
0 & \frac{Z}{R_{0}} & 1+\frac{Y}{R_{0}}
\end{array}\right], \quad[\mathbf{R}] \simeq\left[\begin{array}{ccc}
1 & -\frac{X}{r} & 0 \\
\frac{X}{r} & 1 & -\frac{Z}{R_{0}} \\
0 & \frac{Z}{R_{0}} & 1
\end{array}\right],} \\
{[\mathbf{U}] \simeq\left[\begin{array}{ccc}
1-\frac{Y}{r} & 0 & 0 \\
0 & 1-\frac{Y}{r} & 0 \\
0 & 0 & 1+\frac{Y}{R_{0}}
\end{array}\right]}
\end{array}
$$

${ }_{610}$ Ignoring the infinitesimal terms of higher order than $r^{-1}$ and $R_{0}^{-1}$, it is immediate to check that the tensors 50 satisfy the polar decomposition $\mathbf{F}=$ ${ }_{612}$ RU.

Being $\mathbf{F}=\mathbf{I}+\mathbf{H}$, it is evident from $(50)_{1}$ that the linearized displacement 614 gradient $\mathbf{H}$ is

$$
[\mathbf{H}] \simeq\left[\begin{array}{ccc}
-\frac{Y}{r} & -\frac{X}{r} & 0 \\
\frac{X}{r} & -\frac{Y}{r} & -\frac{Z}{R_{0}} \\
0 & \frac{Z}{R_{0}} & \frac{Y}{R_{0}}
\end{array}\right]
$$

It should be noted that the displacement gradient $\mathbf{H}$ just obtained from the 616 linearized $\mathbf{F}$ coincides with that calculated directly from the infinitesimal displacement field (46).

By applying the theorem of additive decomposition to the linearized displacement gradient $\mathbf{H}$, the skew-symmetric tensor of infinitesimal rigid rotation 
$\mathbf{W}$ and the symmetric tensor of infinitesimal strain $\mathbf{E}$ are obtained ${ }^{13}$

$$
\begin{gathered}
{[\mathbf{W}]=\frac{1}{2}\left[\left(\mathbf{H}-\mathbf{H}^{\mathrm{T}}\right)\right]=\left[\begin{array}{ccc}
0 & -\omega_{z} & \omega_{y} \\
\omega_{z} & 0 & -\omega_{x} \\
-\omega_{y} & \omega_{x} & 0
\end{array}\right] \simeq\left[\begin{array}{ccc}
0 & -\frac{X}{r} & 0 \\
\frac{X}{r} & 0 & -\frac{Z}{R_{0}} \\
0 & \frac{Z}{R_{0}} & 0
\end{array}\right],} \\
{[\mathbf{E}]=\frac{1}{2}\left[\left(\mathbf{H}+\mathbf{H}^{\mathrm{T}}\right)\right]=\left[\begin{array}{ccc}
\varepsilon_{x} & \frac{1}{2} \gamma_{x y} & \frac{1}{2} \gamma_{x z} \\
\frac{1}{2} \gamma_{x y} & \varepsilon_{y} & \frac{1}{2} \gamma_{y z} \\
\frac{1}{2} \gamma_{x z} & \frac{1}{2} \gamma_{y z} & \varepsilon_{z}
\end{array}\right] \simeq\left[\begin{array}{ccc}
-\frac{Y}{r} & 0 & 0 \\
0 & -\frac{Y}{r} & 0 \\
0 & 0 & \frac{Y}{R_{0}}
\end{array}\right] .}
\end{gathered}
$$

Among components of the tensor $\mathbf{W}$, the rotation $\alpha$ around the $X$ axis and $\beta$

$$
\begin{aligned}
& \Delta H=\int_{-\frac{H}{2}}^{\frac{H}{2}} \varepsilon_{y} d Y=-\frac{1}{r} \int_{-\frac{H}{2}}^{\frac{H}{2}} Y d Y=0, \\
& \Delta A=\int_{A}\left(\varepsilon_{x}+\varepsilon_{y}\right) d A=-\frac{2 B}{r} \int_{-\frac{H}{2}}^{\frac{H}{2}} Y d Y=0, \\
& \Delta V=\int_{V}\left(\varepsilon_{x}+\varepsilon_{y}+\varepsilon_{z}\right) d V=-\frac{L B}{R_{0}} \int_{-\frac{H}{2}}^{\frac{H}{2}} Y d Y=0 .
\end{aligned}
$$

Alternatively, the linearization of (33), (34) and (36) yield: $H^{\prime}=H, A^{\prime}=B H$ and $V^{\prime}=B H L$, respectively ${ }^{14}$. With the linearization, the Piola-Kirchhoff stress tensor 15 becomes

$$
\left[\mathbf{T}_{R}\right]=\left[\begin{array}{ccc}
\mathrm{S} \cos \frac{X}{r} & -\mathrm{S} \sin \frac{X}{r} & 0 \\
\mathrm{~S} \sin \frac{X}{r} \cos \frac{Z}{R_{0}} & \mathrm{~S} \cos \frac{X}{r} \cos \frac{Z}{R_{0}} & -\mathrm{S}_{Z} \sin \frac{Z}{R_{0}} \\
\mathrm{~S} \sin \frac{X}{r} \sin \frac{Z}{R_{0}} & \mathrm{~S} \cos \frac{X}{r} \sin \frac{Z}{R_{0}} & \mathrm{~S} Z \cos \frac{Z}{R_{0}}
\end{array}\right] \simeq\left[\begin{array}{ccc}
\mathrm{S} & 0 & 0 \\
0 & \mathrm{~S} & 0 \\
0 & 0 & \mathrm{~S}_{Z}
\end{array}\right],
$$

${ }^{13}$ After linearization, the following relationships hold: $\mathbf{R}=\mathbf{I}+\mathbf{W}, \mathbf{U}=\mathbf{I}+\mathbf{E}$.

${ }^{14}$ Using the Taylor series expansions, the following approximation is employed:

$$
\sinh \frac{H}{2 r} \simeq \frac{H}{2 r}+o\left(r^{-2}\right),
$$

as well as similar expressions for different arguments of hyperbolic sine function. 
where 15

$$
\begin{aligned}
& \mathrm{S}=2 \lambda\left[a+b\left(\lambda^{2}+\lambda_{Z}^{2}\right)+c \lambda^{2} \lambda_{Z}^{2}\right]-\frac{d}{\lambda} \simeq 4 Y\left[-\frac{a+3 b+2 c}{r}+\frac{b+c}{R_{0}}\right], \\
& \mathrm{S}_{Z}=2 \lambda_{Z}\left[a+2 b \lambda^{2}+c \lambda^{4}\right]-\frac{d}{\lambda_{Z}} \simeq-4 Y\left[\frac{2(b+c)}{r}+\frac{a+2 b+c}{R_{0}}\right] .
\end{aligned}
$$

632

The linearized Piola-Kirchhoff stress state is triaxial and principal, it depends (linearly) only on the variable $Y$ and vanishes for $Y=0$. The linearization of the Cauchy stress components 41 gives ${ }^{16}$

$$
\begin{aligned}
& \mathrm{T}_{1}=\mathrm{T}_{2} \simeq 4 Y\left(-\frac{a+3 b+2 c}{r}+\frac{b+c}{R_{0}}\right), \\
& \mathrm{T}_{3} \simeq 4 Y\left[-\frac{2(b+c)}{r}+\frac{a+2 b+c}{R_{0}}\right],
\end{aligned}
$$

showing how these stress components are equal to components 56 . In fact, as ${ }^{36}$ is well known, in the infinitesimal theory the Piola-Kirchhoff and Cauchy stress measures coincide. Moreover, the neutral axis of strain coincides with that of the stress and pass through the centroid of the cross section.

The linearization of the four expressions, obtained from the calculus of integrals 444, provides

$$
\begin{aligned}
& 2 a\left(1+\frac{r}{R_{0}}\right) B H-8 a \frac{r^{3}}{R_{0}} \sinh \left(\frac{H}{2 r}\right) \sin \left(\frac{B}{2 r}\right) \simeq 2 a B H, \\
& 4 b\left(1+\frac{r}{R_{0}}\right) r B \sinh \left(\frac{H}{r}\right)-\frac{16}{3} b \frac{r^{3}}{R_{0}} \sinh \left(\frac{3 H}{2 r}\right) \sin \left(\frac{B}{2 r}\right) \simeq 4 b B H, \\
& c\left(1+\frac{r}{R_{0}}\right) r B \sinh \left(\frac{2 H}{r}\right)-\frac{8}{5} c \frac{r^{3}}{R_{0}} \sinh \left(\frac{5 H}{2 r}\right) \sin \left(\frac{B}{2 r}\right) \simeq 2 c B H, \\
& -(2 a+4 b+2 c) \frac{r R_{0}}{r+R_{0}}\left\{\frac{B H}{r}-B \ln \left[\frac{r+R_{0}-r e^{\frac{H}{2 r}}}{r+R_{0}-r e^{-\frac{H}{2 r}}}\right]\right. \\
& \left.\quad+\frac{\left(r+R_{0}\right) B^{3} \sinh \left(\frac{H}{2 r}\right)}{12 r\left[2 r\left(r+R_{0}\right) \cosh \left(\frac{H}{2 r}\right)-\left(2 r^{2}+2 r R_{0}+R_{0}^{2}\right)\right]}\right\} \simeq-(2 a+4 b+2 c) B H .
\end{aligned}
$$

${ }^{15}$ Using the Taylor series expansions, the following approximation is employed:

$$
\frac{1}{\lambda} \simeq 1+\frac{Y}{r}+o\left(r^{-1}\right),
$$

and the relationship among the constitutive constants $(9)$ has been used to obtain ( 56 .

${ }^{16}$ Using the Taylor series expansions, the following approximation is employed:

$$
\frac{1}{\lambda^{2} \lambda_{Z}} \simeq 1+\frac{2 Y}{r}-\frac{Y}{R_{0}}+o\left(r^{-1}\right)+o\left(R_{0}^{-1}\right) .
$$


The sum of these four terms shows that the normal force $n$ in the linearized

$$
\begin{aligned}
& 2 a\left(1+\frac{r}{R_{0}}\right) r B H-8 a\left(1+\frac{2 r}{R_{0}}\right) r^{3} \sinh \left(\frac{H}{2 r}\right) \sin \left(\frac{B}{2 r}\right) \\
& +a \frac{r^{4}}{R_{0}} \sinh \left(\frac{H}{r}\right)\left[\sin \left(\frac{B}{r}\right)+\frac{B}{r}\right] \simeq \frac{a}{12 p R_{0}}\left[(2 p-1) B H^{3}+B^{3} H\right], \\
& 4 b\left(1+\frac{r}{R_{0}}\right) r^{2} B \sinh \left(\frac{H}{r}\right)-\frac{16}{3} b\left(1+\frac{2 r}{R_{0}}\right) r^{3} \sinh \left(\frac{3 H}{2 r}\right) \sin \left(\frac{B}{2 r}\right)+ \\
& \quad b \frac{r^{4}}{R_{0}} \sinh \left(\frac{2 H}{r}\right)\left[\sin \left(\frac{B}{r}\right)+\frac{B}{r}\right] \simeq \frac{2 b}{12 p R_{0}}\left([2 p-5] B H^{3}+B^{3} H\right), \\
& c\left(1+\frac{r}{R_{0}}\right) r^{2} B \sinh \left(\frac{2 H}{r}\right)-\frac{8}{5} c\left(1+\frac{2 r}{R_{0}}\right) r^{3} \sinh \left(\frac{5 H}{2 r}\right) \sin \left(\frac{B}{2 r}\right)+ \\
& \frac{c}{3} \frac{r^{4}}{R_{0}} \sinh \left(\frac{3 H}{r}\right)\left[\sin \left(\frac{B}{r}\right)+\frac{B}{r}\right] \simeq \frac{c}{12 p R_{0}}\left([2 p-9] B H^{3}+B^{3} H\right), \\
& -(2 a+4 b+2 c)\left\{\frac{r R_{0} B}{r+R_{0}}\left[H-R_{0} \ln \left(\frac{r+R_{0}-r e^{-\frac{H}{2 r}}}{r+R_{0}-r e^{\frac{H}{2 r}}}\right)\right]\right. \\
& \left.-\frac{R_{0}^{2} B^{3}\left(e^{\frac{H}{r}}-1\right)}{24\left[\left(e^{\frac{H}{2 r}}-1\right) r-R_{0}\right]\left[\left(e^{\frac{H}{2 r}}-1\right) r+R_{0} e^{\frac{H}{2 r}}\right]}\right\} \\
& \simeq \frac{a+2 b+c}{12 p R_{0}}\left([2 p+1] B H^{3}-B^{3} H\right),
\end{aligned}
$$

where, using 23 with $p=\frac{a+3 b+2 c}{b+c}$, the radius $r$ has been replaced with the

radius $R_{0}$. The sum of these four terms gives the linearized moment-curvature relationships

$$
m_{x}=\frac{4(a+b)(a+4 b+3 c)}{a+3 b+2 c} \frac{B H^{3}}{12} \frac{1}{R_{0}} .
$$

648

It is important to note that the linearized stresses $(56)$, or equivalently the (57), differ from the corresponding stress components of linear theory. This is compressible Mooney-Rivlin law in nonlinear theory and the Navier inverse law in linear theory

$$
\overline{\mathbf{T}}=2 G \mathbf{E}+\bar{\lambda}(\operatorname{tr} \mathbf{E}) \mathbf{I},
$$

where $G$ and $\bar{\lambda}$ are the Lamé constants and $\operatorname{tr} \mathbf{E}=\varepsilon_{x}+\varepsilon_{y}+\varepsilon_{z}$. The diagonal 654 components of $\overline{\mathbf{T}}$ are denoted by $\sigma_{x}, \sigma_{y}, \sigma_{z}$. However, certain conditions can be 
established for the constitutive parameters $a, b$ and $c$ of a compressible Mooney-

Rivlin material in order to reproduce the same stresses of the classical linear theory. Equating the component $\sigma_{x}=2 G \epsilon_{x}+\bar{\lambda}\left(\varepsilon_{x}+\varepsilon_{y}+\varepsilon_{z}\right)$ of 61 with the linearized expression $561_{1}$ of $S$, with $-\frac{Y}{r}=\epsilon_{x}=\epsilon_{y}$ and $\frac{Y}{R_{0}}=\epsilon_{z}$ according to (53), the following relations are obtained:

$$
\left\{\begin{array}{l}
G=2(a+b) \\
\bar{\lambda}=4(b+c)
\end{array}\right.
$$

6ro Lamé constants $G$ and $\bar{\lambda}$ can be then converted into the pair of elastic constants $E$ and $\nu$

$$
\left\{\begin{array}{l}
E=\frac{G(3 \bar{\lambda}+2 G)}{\bar{\lambda}+G}=\frac{4(a+b)(a+4 b+3 c)}{a+3 b+2 c} \\
\nu=\frac{\bar{\lambda}}{2(\bar{\lambda}+G)}=\frac{b+c}{a+3 b+2 c}
\end{array} .\right.
$$

${ }_{62}$ Introducing the strain tensor $\mathbf{E}$, in the form specified by $(53)$, into the constitutive law 61), remembering that $R_{0}=\nu r$ and using relations 63 , the stress tensor $\overline{\mathbf{T}}$ assumes the following form:

$$
[\overline{\mathbf{T}}]=\left[\begin{array}{ccc}
0 & 0 & 0 \\
0 & 0 & 0 \\
0 & 0 & E \varepsilon_{z}
\end{array}\right]
$$

showing, as is well known, that in the linearized theory the stress state is uniaxial being different from zero only the component $\sigma_{z}{ }^{17}$

Finally, it can be observed that, replacing $(63)_{2}$ into $(23)$, the relationship $47)_{2}$ is recovered. Moreover, being $J_{X}=\frac{B H^{3}}{12}$, the substitution of 63 into $_{1}$ into 60) provides the linear moment-curvature relation 47$]_{1}$.

\footnotetext{
${ }^{17}$ Of course, the same result can be achieved for a compressible Mooney-Rivlin material that satisfies the conditions 62 . In effect, replacing 63 into 56 , it is found

$$
\begin{aligned}
\mathrm{S} & =\left[-(a+3 b+2 c)+\frac{b+c}{\nu}\right] \frac{4 Y}{r}=0, \\
\mathrm{~S}_{Z} & =[-2(b+c) \nu+(a+2 b+c)] \frac{4 Y}{R_{0}}=E \varepsilon_{z} .
\end{aligned}
$$
}




\subsection{Numerical analysis}

674 merical simulations have been carried out. The approach is aimed to model

With these last observations, the linearization procedure which leads from the finite to the infinitesimal bending of a beam is completed.

\section{The numerical and experimental analyses}

numerically the flexion of the reference beam under large deformations and displacements.

In order to reduce the computational effort, only one half beam has been considered ( $c f$. Figure 20(a) . Two different types of restraints have been used

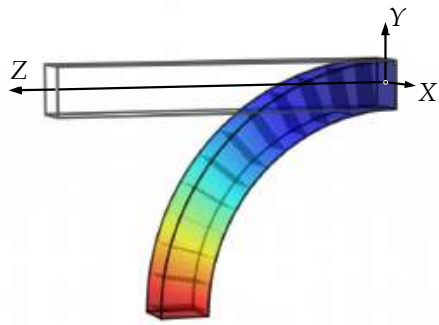

(a) Numerical model. Deformed configuration for $\alpha_{0}=\pi / 2$.

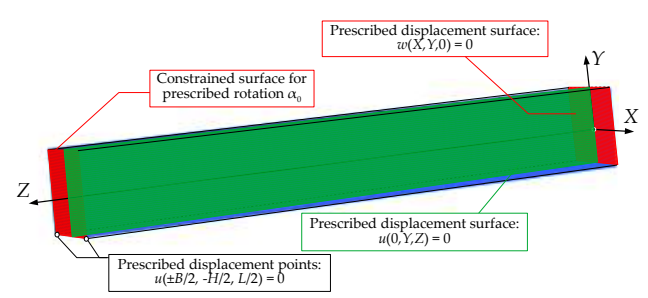

(b) FE mesh. Assignment of the constraints.
Figure 20: Details about the FE model. symmetry condition, the displacement component $w(X, Y, 0)$ in the $X Y$ plane 2 transversal inflexion which characterizes the anticlastic effect. Furthermore, the $Z Y$ middle longitudinal section of the specimen has been restrained in terms of the displacement component $u(0, Y, Z)$. Finally, the rigid body mo86 tion is avoided simply by pinning the lower nodes of the middle cross section at $( \pm B / 2,-H / 2,0)$, thus preventing their vertical displacement component $v(c f$. 


\footnotetext{
${ }^{18}$ This rigid constraint allows reproducing the experimental set-up, described in Section 3.2 where the ends of the beam are glued to the metallic plates of the mechanical apparatus.

${ }^{19}$ The imparting the flexure angle $2 \alpha_{0}$ to the beam ends with the FE approach deserves some difficulties. To this aim, a distribution of stress should be applied at the two end cross sections in order to reproduce a constant bending moment along the longitudinal axis of the beam. As an alternative, a displacement field could be imposed at the end cross sections. Based on the assumption of planarity of the cross sections, this should produce a deformed configuration resembling that a circumferential arc. However, in both situations, stresses and displacements to be applied depend on the solution of the equilibrium problem and they are not known a priori.
} 


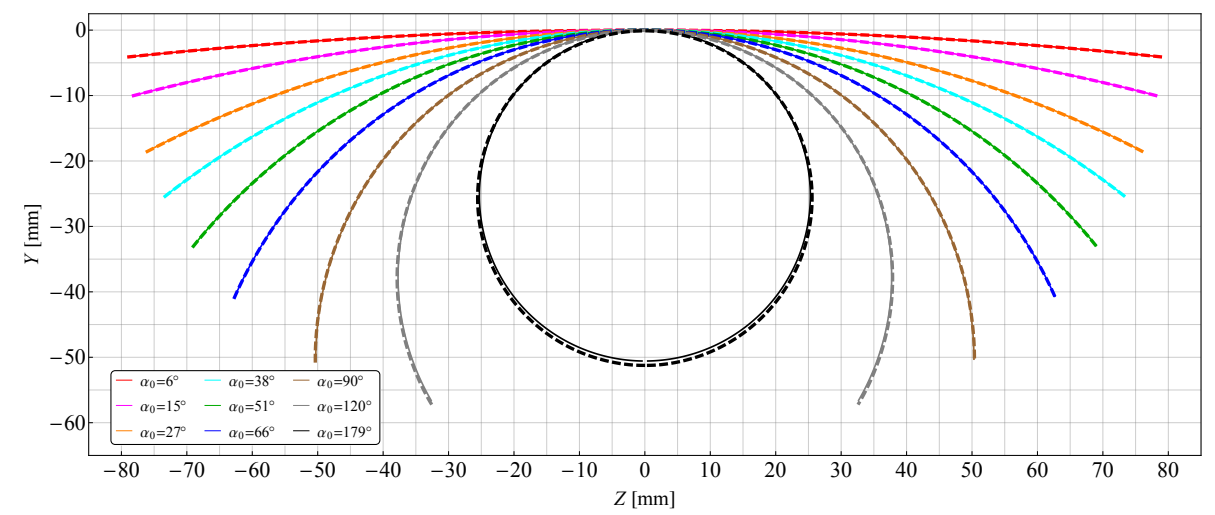

(a) Deformed configuration of the longitudinal centroid line $(X=0, Y=0, Z)$.

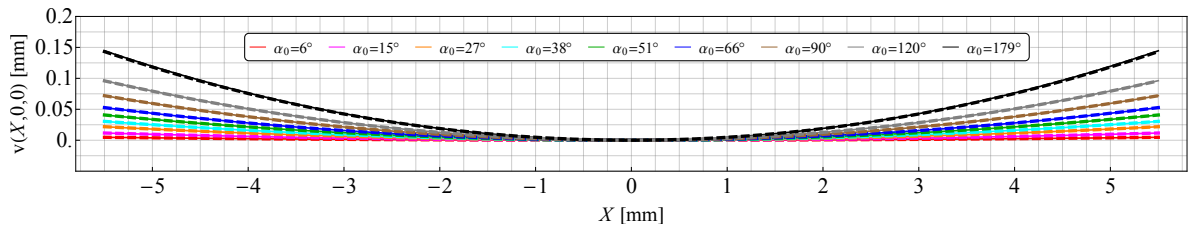

(b) Displacement component $v(0,0, Z)$ of the transversal centroid line $(X Y=0 Z=0)$.

Figure 21: Comparison between the results provided by the numerical (dashed lines) and theoretical (solid lines) models.

$X=X, Y=Z=0$ (cf. Figure 21(b). To assess the gap between the values provided by the theoretical model and those obtained by the numerical analysis, the relative error $\epsilon_{r, d}$ for displacements is introduced, being $\epsilon_{r, d}=\epsilon / v_{T M} 100$, $\epsilon=v_{T M}-v_{F E M}$ (absolute error), $v_{T M}$ is the value furnished by the theoretical model and $v_{F E M}$ that obtained by the FE analysis. Figure 22 shows the maximum errors varying $\alpha_{0}$. In particular, for $\alpha_{0}=179^{\circ}$, at the ends of the beam ( $Z= \pm L / 2)$, the relative error $\epsilon_{r, d}$ reaches the maximum value of about $1.39 \%$. Concerning the transversal displacement field reported in Figure 21(b) (strictly connected to the anticlastic effect), for $\alpha_{0}=179^{\circ}, \epsilon_{r, d}$ reaches the maximum value of about $0.92 \%$ at the beam lateral surface, namely at $X= \pm B / 2,(c f$. 20 Figure 22.

For the limit case with $\alpha_{0}=179^{\circ}$, that is for a beam deformed like a ring, 722 Figure 23 shows the stretches $\lambda_{Z}$ for each point of the middle cross section 


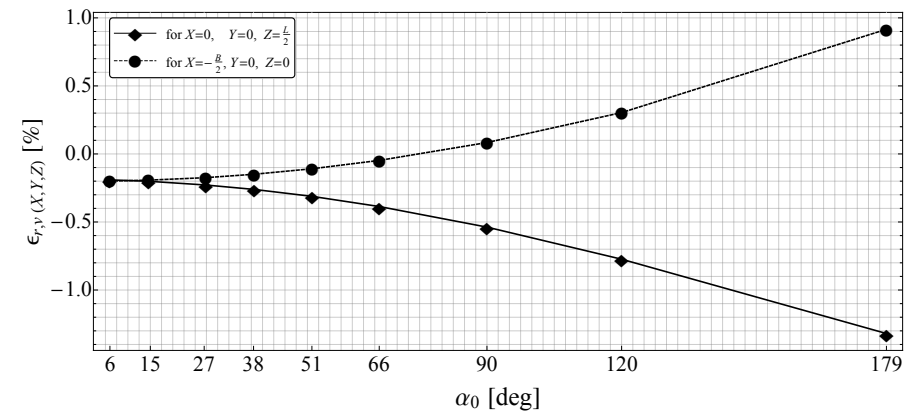

Figure 22: Relative error affecting the vertical displacements varying the angle $\alpha_{0}$. Vertical displacements $v(0,0, L / 2)$ (solid line) and $v(-B / 2,0,0)$ (dashed line).

$(Z=0)$, evaluated both in the reference and spatial configurations ( $c f$. Figures 23(a) and 23(b)]. Dashed iso-lines in the contour plot denote the results

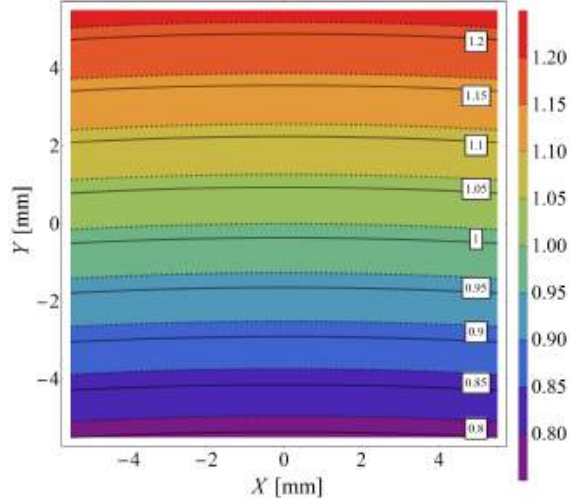

(a) Lagrangian stretch $\lambda_{Z}(X, Y, Z)$.

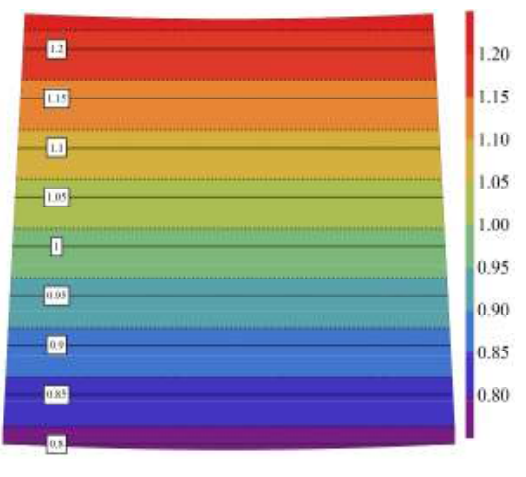

(b) Eulerian stretch $\lambda_{z}(x, y, z)$.

Figure 23: Numerical (solid iso-lines with framed value) and theoretical (contour-plot with dotted iso-lines) results for both the reference and deformed configurations. 


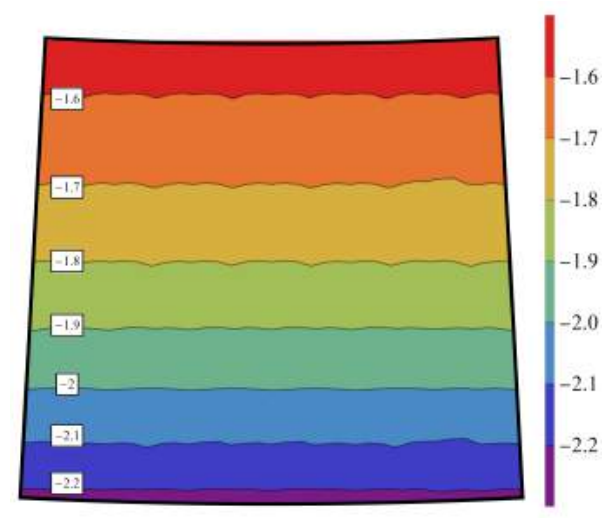

Figure 24: Relative errors (experimental with respect to the theoretical) about the Eulerian stretch $\lambda_{z}(x, y, z)$.

${ }_{730}$ are displayed ${ }^{20}$. The major gap occurs at the bottom edge, where the FE analysis slightly overestimates the results of the theoretical model by a few percentage points.

Figure 25 shows the comparisons in terms of longitudinal stresses. In particular, Figure 25(a) highlights the Piola-Kirchhoff stress component $\mathrm{T}_{R, 33}$, whereas the Cauchy stress component $\mathrm{T}_{33}$ is plotted in Figure 25(b). The comparison shows slight differences between the results obtained with the two approaches. The absolute errors concerning the Cauchy stress component $\left(\epsilon=\mathrm{T}_{33, T M^{-}}\right.$ $\left.{ }_{738} \mathrm{~T}_{33, F E M}\right)$ are shown in Figure 2(101

\subsection{Experimental analysis}

740

An experimental analysis for the bending of the reference beam is carried out in this Section and the obtained results are compared with those provided by the theoretical model. Recently, a proper mechanical prototype (see Figure 28) has been designed and manufactured for this type of experimental investigation

\footnotetext{
${ }^{20}$ Oscillations in the iso-lines are due to the fact that the numerical results have been assessed at the Gauss nodes of the FE mesh.

${ }^{21}$ Note that the relative errors for stresses become meaningless in the central area of the cross section as the stresses vanish there.
} 


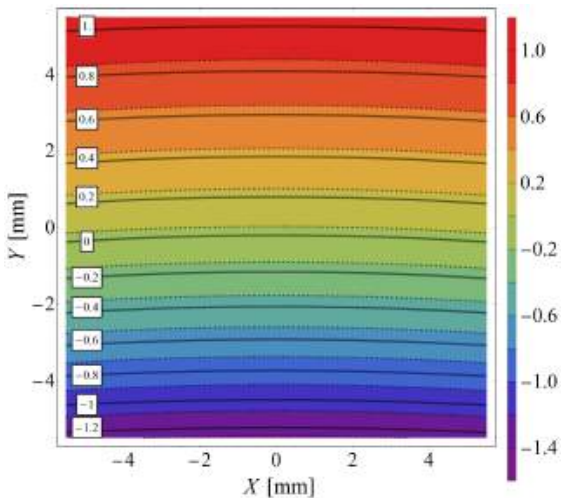

(a) Lagrangian (Piola-Kirchhoff) stress component $\mathrm{T}_{R, 33}(X, Y, 0)$.

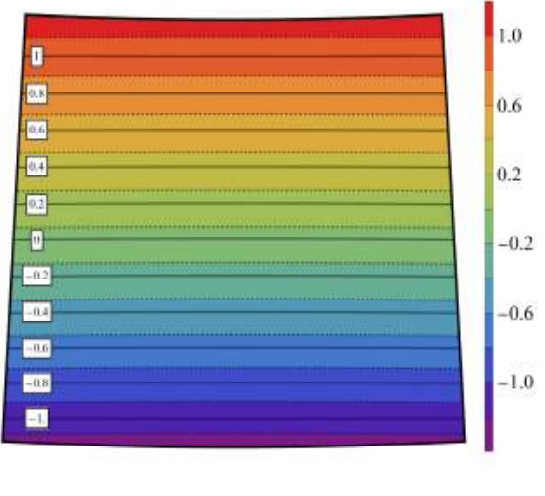

(b) Eulerian (Cauchy) stress component $\mathrm{T}_{33}(x, y, 0)$.

Figure 25: FE (solid iso-lines with framed value) and theoretical (contour-plot with dotted iso-lines) results for both the reference and deformed configurations of the beam cross section at $Z=0$.

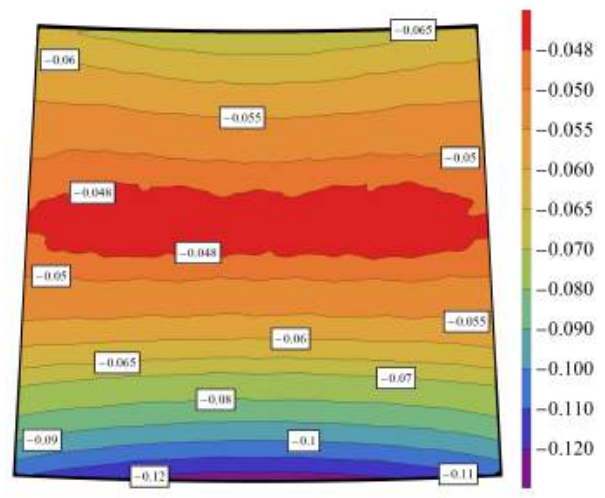

Figure 26: Absolute error (experimental with respect to the theoretical) of the Cauchy stress component $\mathrm{T}_{33}(x, y, 0)$.

30. Concerning the realization of this test equipment, the main difficulty is to reproduce a pure bending state avoiding unwanted effects, as the occurrence of axial or shear forces. The proposed mechanical apparatus induces bending by imposing the prescribed rotations at the ends of the sample, but letting it free 
to exhibit its elastic retaining forc $e^{22}$

The large bending of the sample is achieved by two pantographs. The two pairs of arms of each pantograph are constrained, in a bilateral manner, to slide on two horizontal guides. These guides are connected by a threaded vertical bar, fixed to the lower guide. The rotation of the activation handle generates a vertical motion of the lower guide approaching (or moving away from) the upper one. The mutual vertical translation of the horizontal guides actuates the scissor devices which imparts a rotation to the slanted arms around their fulcrum. The slanted arms of each pantograph are connected by five hinges and they rotate at each turn of the activation knob. A rigid block is fixed to the upper internal hinge. A little plate with a L-shaped profile is constrained on this block. The end of the sample is glued to the plate. Knowing the thread pitch of the manual actuation device, it is possible to assess exactly the rotation angle $\alpha_{0}$ imparted at the ends of the specimen. The nonlinear correspondence between the number of revolutions of the manual actuation device and the angle $\alpha_{0}$ has been accurately estimated. The number of rotations of the activation handle necessary to produce some values of the angle $\alpha_{0}$ are reported in Figure 27. Such values have been assessed through a numerical algorithm and, experimentally, by measuring the rotation of the rigid block with the digital image correlation (DIC) apparatus.

A DIC instrumentation, a full-field image analysis method based on grey value digital images optical monitoring, has been used to acquire the experimental data, with specific reference to the displacement field. In particular, Istra Q-400 instrumentation of the Dantec Dynamic s.b.m., with its own internal hardware and software equipments, has been used. The calibration of the optical system ensures a monitoring resolution of $\pm 10 \mu \mathrm{m}$. By setting the acquisition procedure in stereo-mode, the entire three-dimensional displacement

\footnotetext{
${ }^{22}$ It should be remarked that the inevitable frictions of the mechanisms during the experimental tests run against to the elastic retaining force. This leads to an overestimation of the stiffness of the tested sample.
} 


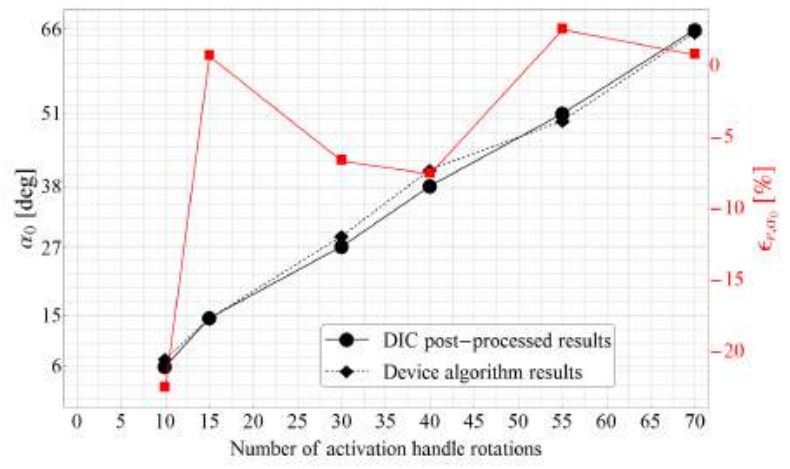

Figure 27: Rotation angle $\alpha_{0}$ monitored with DIC (solid line) and calculated analytically (dashed line) varying the activation handle of the mechanical device.

field of the external surface of the specimen can be assessed. Two different acquisition layouts have been performed: The first set-up, named in the following upper view, consists in the acquisition over the upper-surface of the specimen, that is the horizontal plane $Y=H / 2$; the second acquisition set-up, named lateral view, consists in the lateral monitoring of the specimen on the vertical plane $X=B / 2$. Some details of the upper and lateral views are shown in Figure 29(a), During the bending test, only the two above mentioned surfaces of the specimen are monitored. Some reference lines inside the two monitored surfaces of the sample, along which the line plots are made, are identified as shown in Figure 29(b). Obviously, physical fields inside the beam cannot be measured trough the DIC.

The vertical displacements $v(B / 2,0, Z)$ of the reference line $L_{h}$ varying the angle $\alpha_{0}$ are shown in Figure 30 . The results provided by the experimental analysis are represented with dashed lines, whereas the solid lines denote the results given by the theoretical model. The experimental data and the theoretical predictions are close, showing that the two approaches provide similar results. Note that in the central part of the basic line the two approaches provide almost the same results. For $\alpha_{0}>66^{\circ}$, the specimen is no longer able to develop an effective retaining force, thus the experimental results with $\alpha_{0}>66^{\circ}$ appear less accurate. Note also that in the range of $\alpha_{0}$ here investigated, the 


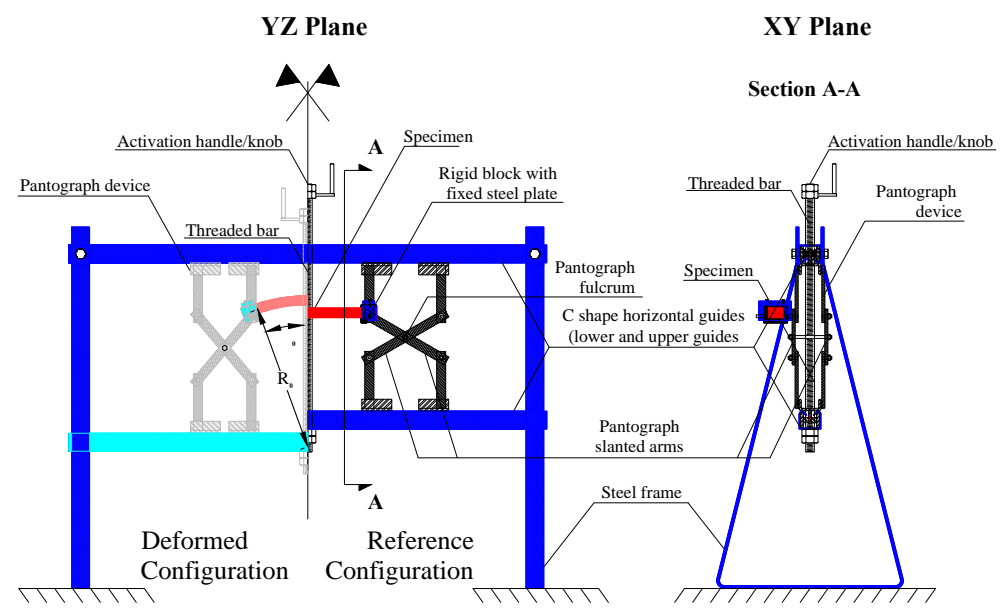

(a) Mechanical schedule.

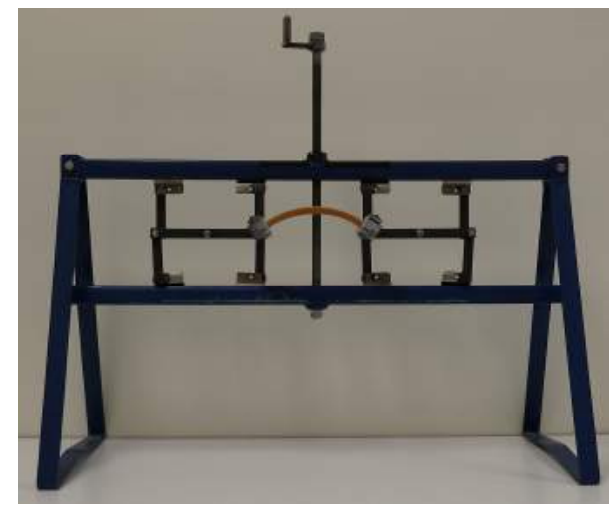

(b) Frontal view.

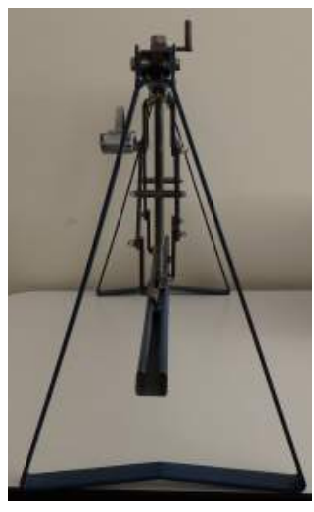

(c) Lateral view.

Figure 28: Mechanical equipment for large bending test.

maximum errors occur at the end cross sections $(Z= \pm L / 2)$.

Figure 31 shows the maximum values of the gap between the two solutions.

For low values of the angle $\alpha_{0}$, significant errors occur owing to the initial frictions of the test machine, that overcome the retaining force of the sample. The iso-lines provided by the experimental tests (solid lines) are close to those obtained from the theoretical prediction.

The deformed configurations assumed by the specimen during the experimental tests are shown in Figure 33 for the first six values assumed by $\alpha_{0}$. The 

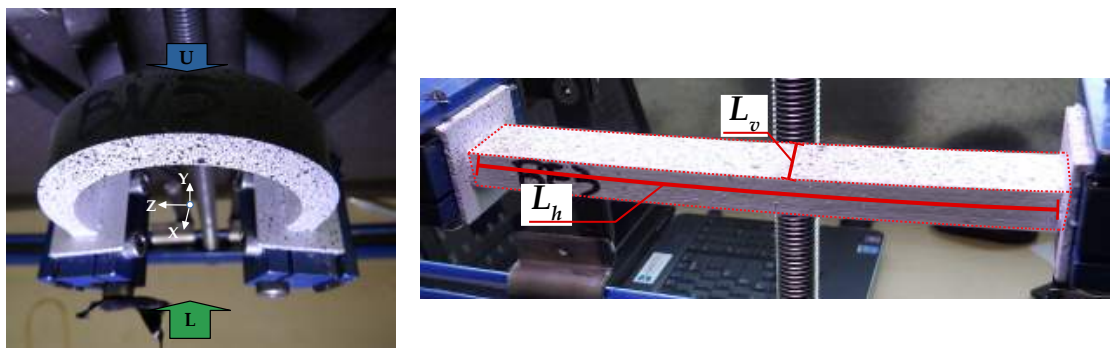

(a) Monitored specimen under (b) Experimental reference line on the monitored specbending: Lateral (L) and upper imen: $L_{v}$ vertical line for the upper monitoring and $L_{h}$ (U) views. horizontal line for the lateral monitoring.

Figure 29: DIC monitoring views and reference lines.

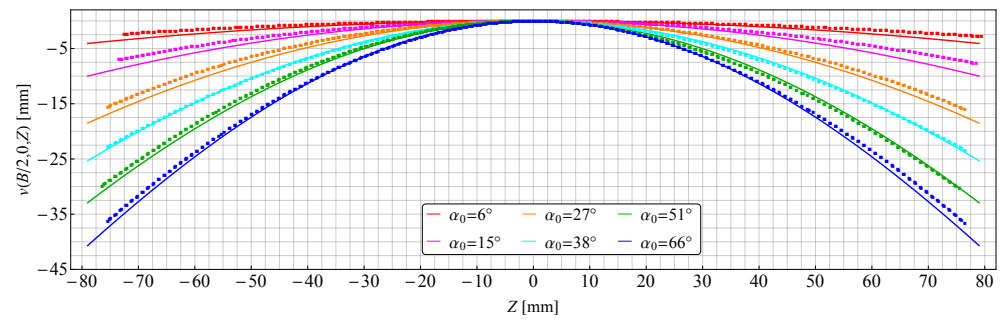

Figure 30: Vertical displacements component $v(B / 2,0, Z)$ along the reference line $L_{h}$ varying the angle $\alpha_{0}$ : Experimental results (dashed lines) and theoretical prediction (solid lines).

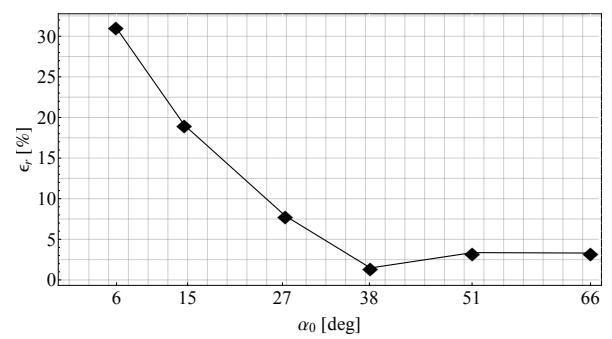

Figure 31: Relative error about the vertical displacements $v(B / 2,0,0)$ (experimental with respect to the theoretical) varying the angle $\alpha_{0}$.

same figure highlights the contour-plots of the rotation field measured on the lateral surface of the sample. As expected from the hypothesis of conservation of the planarity of the cross sections, the iso-lines represented in the reference 


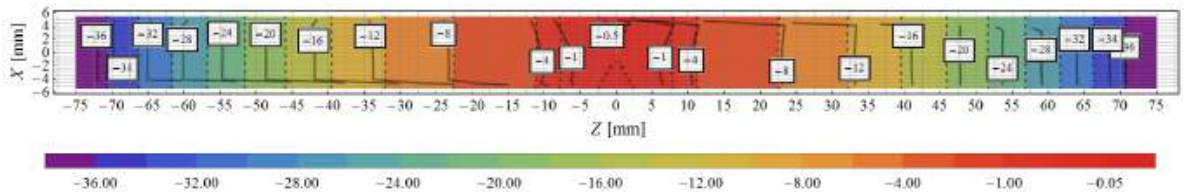

(a) Displacement component $v(X, H / 2, Z)$ at the upper specimen surface.

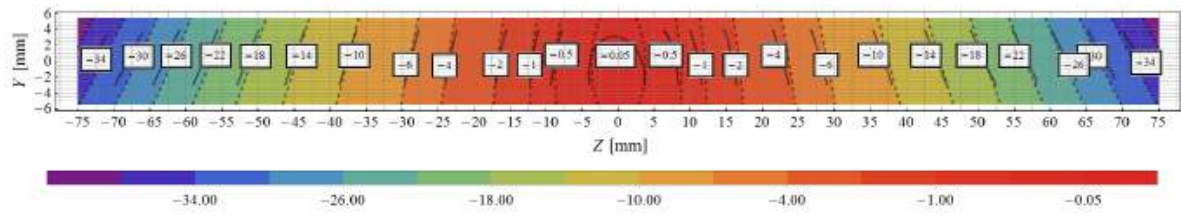

(b) Displacement component $v(B / 2, Y, Z)$ at the lateral specimen surface.

Figure 32: Experimental (solid iso-lines with framed value) and theoretical (contour-plot with dotted iso-lines) results.

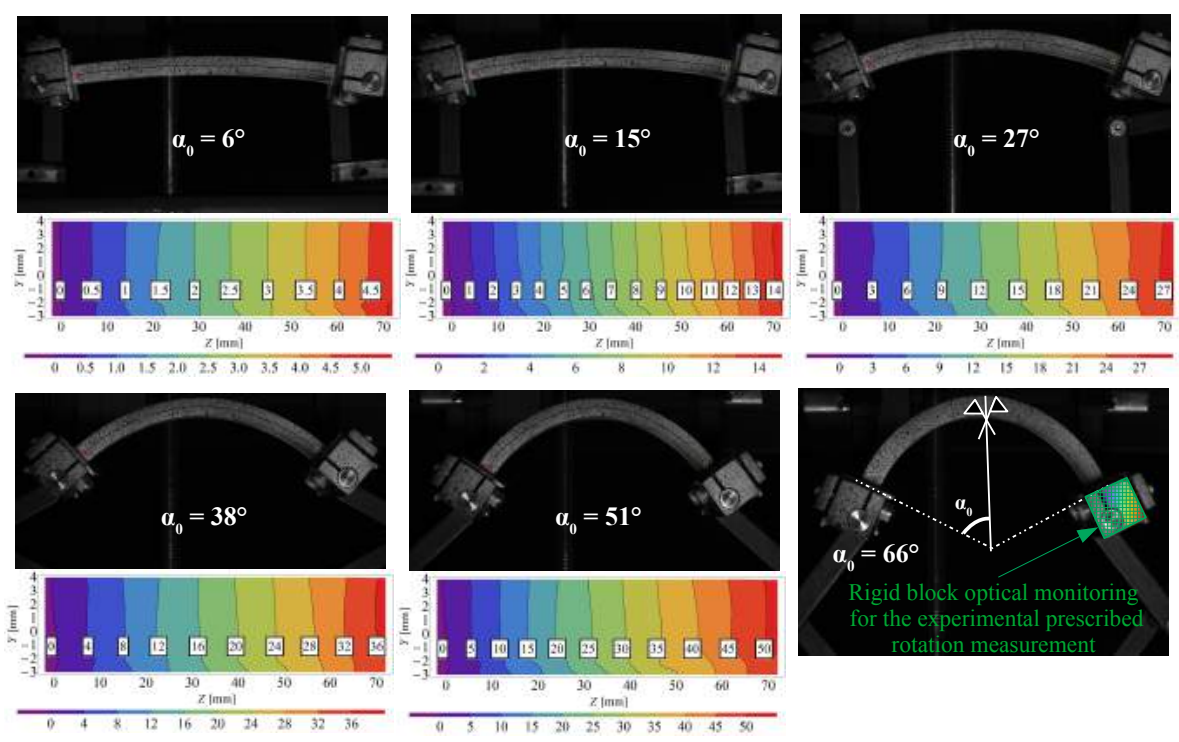

Figure 33: Contour-plot of the rotation field around the $X$ axis in the reference configuration. Acquired DIC data for some values of the angle $\alpha_{0}$.

configuration are equispaced and sufficiently straight 23 .

\footnotetext{
${ }^{23}$ The last image of Figure 33 shows the rigid block that, monitored with the DIC instrument, allows obtaining the rotations shown in Figure 27
} 


\subsection{Discussion}

808 numerical simulations and experimental investigation are compared in Figure 34 As reported in Section 3.2 the experimental results referred to imparted rota-

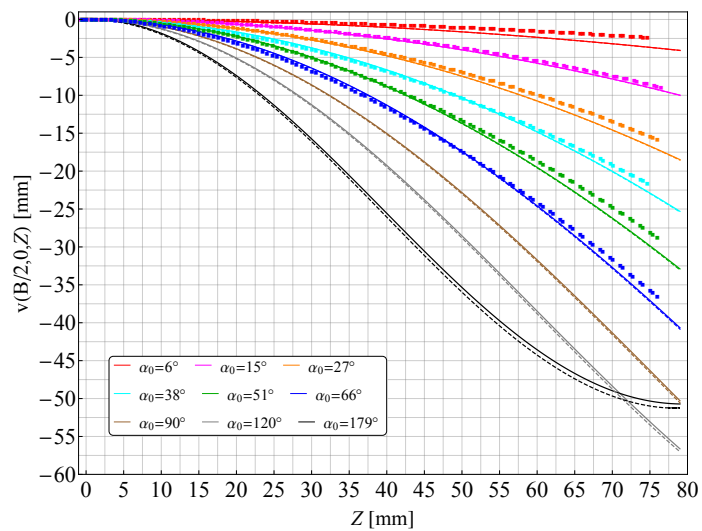

Figure 34: Displacements component $v(B / 2,0, Z)$. Theoretical model (solid lines), numerical analysis (dashed lines) and experimental analysis (cross marker) for $Z \in[0, L / 2]$ varying $\alpha_{0}$.

tions $\alpha_{0} \leq 66^{\circ}$ owing to technical difficulties encountered during the tests. Note 812 that the shape of the curve relative to the case $\alpha_{0}=179^{\circ}$ is different from the others, since the ends of the beam are also pushed upwards to form the ring ( $c f$. Figure 21(a)p. As shown, for the selected values of $\alpha_{0}$, the curves are very close to each other.

With more detail, the rotations for the last case of Figure 33 corresponding to $\alpha_{0}=66^{\circ}$ are illustrated in Figure 35, where the rotation iso-lines of the cross sections are also reported. The amount of rotation undergone by the beam cross sections are shown in Figure $35(\mathrm{a})$ in the reference configuration ${ }^{24}$. Owing to symmetry, the middle cross section does not exhibit rotation, whereas the other cross sections highlighted in the figure correspond to the cross sections that undergo the rotations reported in the black boxes. These cross sections are perfectly equi-spaced each other. As shown, the theoretical model (contour-

\footnotetext{
${ }^{24}$ For sake of representation, the height of the specimen has been doubled.
} 


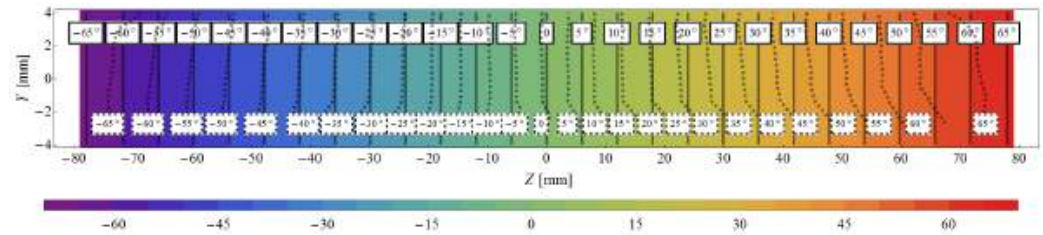

(a) Lateral surface of the specimen in the Lagrangian description.

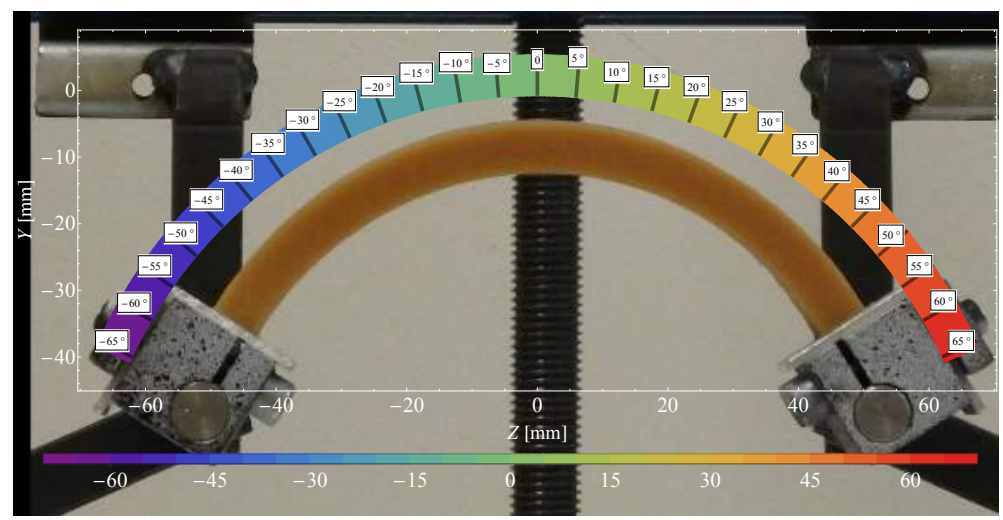

(b) Lateral surface of the specimen in the Eulerian description.

Figure 35: Comparison between the rotation field provided by the theoretical model (contourplot), FE analysis (solid lines) and the experimental analysis (dashed iso-lines, for the reference configuration only) for $\alpha_{0}=66^{\circ}$.

plot) and the FE analysis (continuous iso-lines) provide coincident results, while the results obtained by the experimental analysis (dashed lines) differ a little. 826 Making reference to the Eulerian description, Figure 35(b) shows the same rotations in the deformed configuration. In such figure some technical difficulties were encountered in representing the experimental results, which came out of the profile of the deformed specimen. For this reason, only the results of the theoretical model and those of the FE analysis are compared, which substantially coincide. Note that in Figure $35(\mathrm{~b})$ the vertical traces of the cross sections, positioned on the lateral surface of the specimen, remain rectilinear after the deformation. This confirms the hypothesis of conservation of the planarity of 834 the beam cross sections.

Figure $36(\mathrm{a})$ shows the longitudinal radius $R$ evaluated along the reference 
line $L_{h}$ for $\alpha_{0} \leq 66^{\circ}$. As expected from the theoretical model, this geomet-

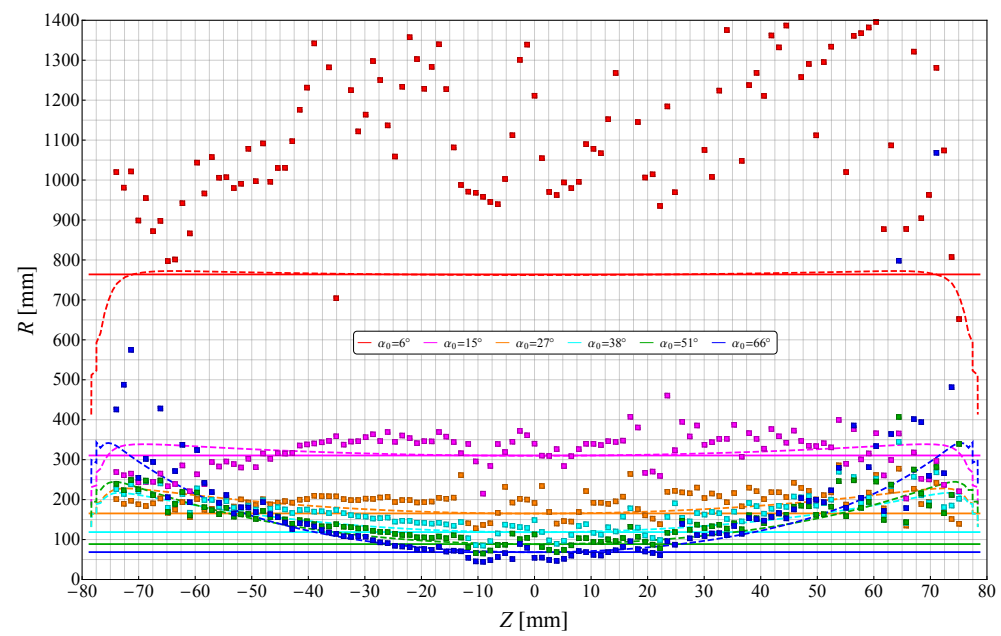

(a) Longitudinal radius of curvature $R$ along the reference line $L_{h}$.

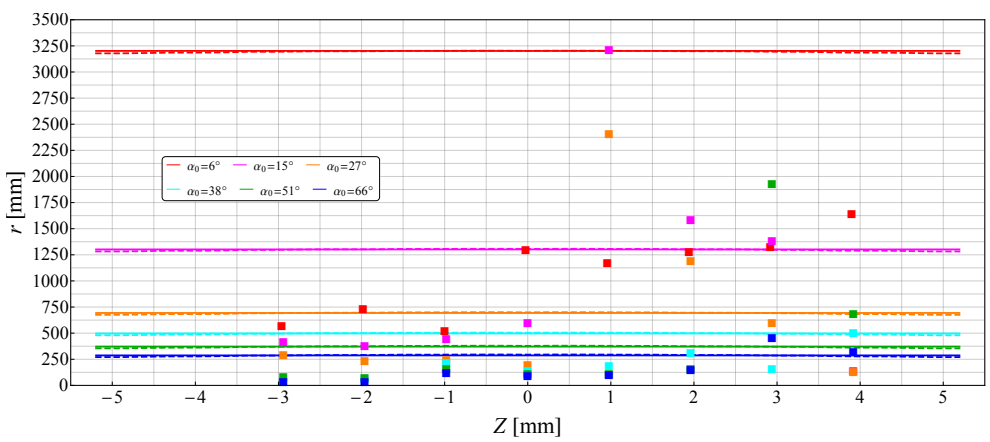

(b) Anticlastic radius of curvature $r$ along the reference line $L_{v}$.

Figure 36: Variation of the radii of curvature along the reference lines: Theoretical model (solid lines), numerical analysis (dashed lines) and experimental analysis (dotted lines with cross marker).

836

ric parameter is constant along the beam axis. Apart the end regions of the beam, the same result is provided by the FE analysis (the noise produced by the imposition of the boundary conditions has been discussed in Section 3.1.

840 Experimental results are represented by a set of discrete points. The spread affecting the experimental values for $\alpha_{0}=6^{\circ}$ is due to the initial friction of the test machine. As expected, as the angle $\alpha_{0}$ increases, the radius $R$ decreases 
and the experimental values converge to the theoretical and numerical results, showing a constant trend along the beam axis. In Figure 36(b), the results of the three different methods are compared in terms of the anticlastic radius $r$ for the reference line $L_{v}$ at the middle cross section. For each value of the angle $\alpha_{0}$, the theoretical model predicts a constant transverse radius $r$. The FE analysis provides the same constant values along the transversal direction ${ }^{25}$ Conversely, as discussed in Section 3.2 , the results provided by the experimental analysis are not very reliable due to the difficulty of measuring displacements of small amount.

To assess in detail the transversal radius of curvature $r$ at each point of the middle cross section, a specific FE analysis was carried out ${ }^{26}$ The values of $r$ 854 for $\alpha_{0}=90^{\circ}$ and $\alpha_{0}=179^{\circ}$ are shown in Figures 37(a) and 37(b), respectively. It is remarked that the theoretical model assumes a single value of $r$ for all

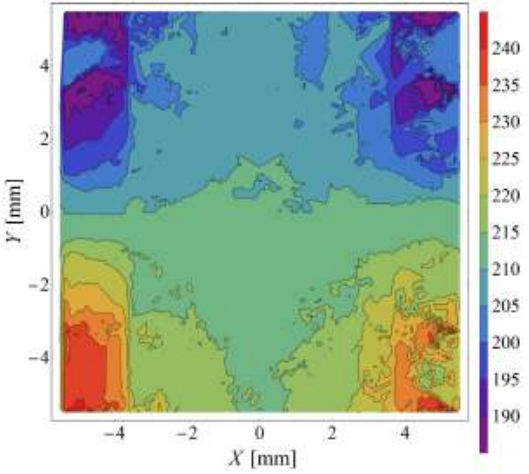

(a) Values of $r$ for $\alpha_{0}=90^{\circ}$.

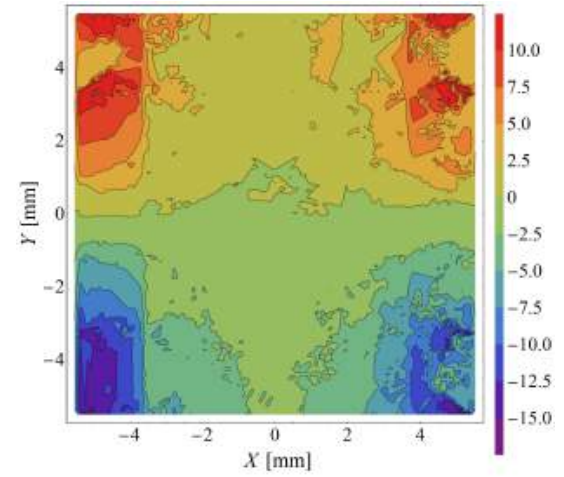

(b) Values of $r$ for $\alpha_{0}=179^{\circ}$.

Figure 37: Transversal radius of curvature $r$ provided by the FE analysis for each point of the middle cross section.

points of the transversal basic line $X=X$ and $Y=Z=0$, coinciding with

\footnotetext{
${ }^{25}$ The FE results in the middle section are not affected by the discrepancies generated by the boundary conditions.

${ }^{26}$ The radius $r$ has been evaluated locally as the radius of the osculating circle passing through three neighbouring points of the reference line in the deformed configuration.
} 
the value of $r$ at the centroid, $r_{T M}$. As it can be seen in Figure 37, such an clear) together with the profile assumed by the transversal basic line, which assumes the radius of curvature $r=r_{T M}$. The distribution of the longitudinal

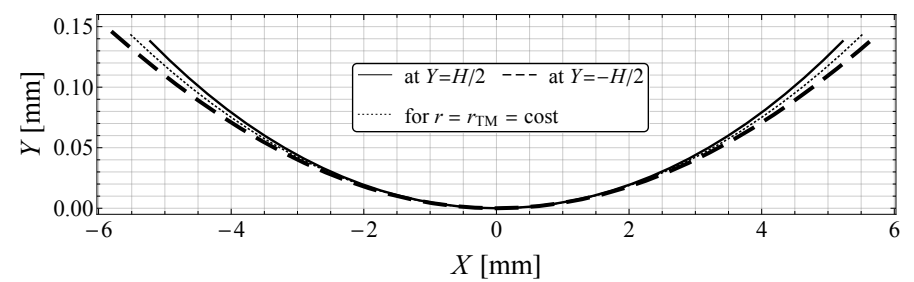

Figure 38: Deformed configuration of the upper (solid line) and lower (dashed line) edges of the middle cross section for $\alpha_{0}=179^{\circ}$ provided by the $\mathrm{FE}$ analysis.

874

stretches $\lambda_{z}(0, y, 0)$ in the Eulerian configuration varying $\alpha_{0}$ are shown in Fig-

Further considerations on the trend of $r$ within the cross section can be made from Figures 37. In particular, the radius $r$ varies slightly inside the core of the cross section, assuming locally values close to $r_{T M}$. Instead, significant differences occur in the correspondence of the four vertices of the cross section. This is due to the fact that vertices are far from the transversal basic line. thus keeping a certain capacity to deform. The edge of the beam cross section near the upper vertices tends to form curls and therefore $r$ is lower than $r_{T M}$ there. Conversely, the edge of the cross section near the lower vertices tends to become straight, and therefore in these zones $r$ is larger than $r_{T M}$. Figure 38 shows the deformed configurations assumed by the upper and lower edges of the middle cross section for $\alpha_{0}=179^{\circ}$ (the amplitude of the in-plane displacement components $u(X, \pm H / 2,0)$ and $v(X, \pm H / 2,0)$ has been magnified and the deformed profiles overlapped at $X=0$ to make the image more ure 39(a) Based on the theoretical prediction, the longitudinal stretches exhibit a linear variation along the $y$ axis, assuming unitary value at the origin. Such linear trend do not depend on the Eulerian variable $x$. Therefore, the vertical diagram of stretches remains unchanged moving transversely in the $x y$ plane. 


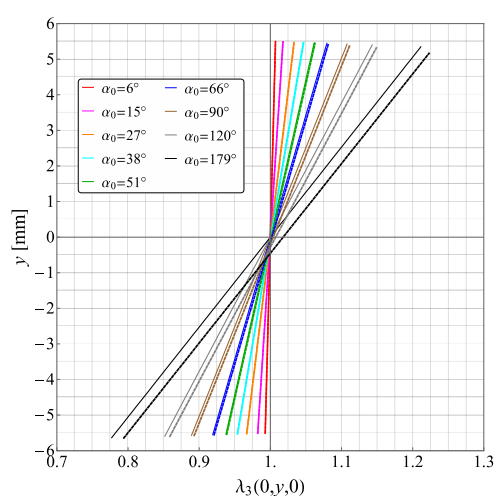

(a) Vertical diagrams of $\lambda_{z}(0, y, 0)$ varying $\alpha_{0}$. Comparison between theoretical (solid lines) and FE (dashed lines) results.

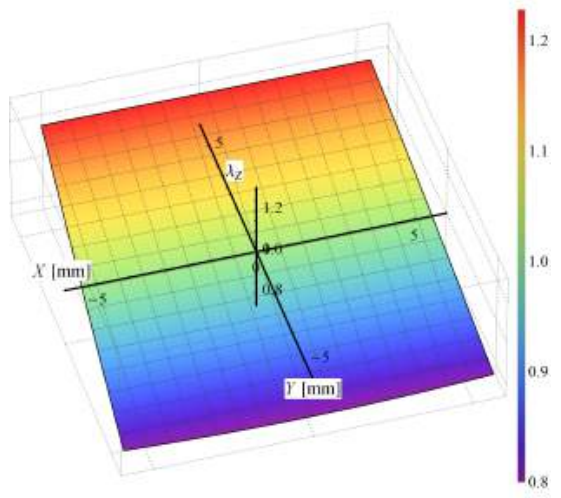

(b) 2D diagram of $\lambda_{z}(x, y, 0)$ for $\alpha_{0}=179^{\circ}$ provided by FE analysis.

Figure 39: Longitudinal stretches $\lambda_{z}$ at the middle cross section.

In particular, all stretches $\lambda_{z}(x, 0,0)$ evaluated along the $x$ axis are unitary. As a consequence, $x$ axis coincides with the neutral axis for the deformation (cf. Figure 39(b)). Such considerations about stretches are consequences of the hypothesis of conservation of the planarity of the cross sections. The results obtained by the theoretical model discussed above are substantially confirmed by the numerical ones provided by FE analysis.

\section{Conclusions}

In this paper, the equilibrium problem of hyperelastic slender beams under anticlastic bending has been investigated in the context of finite elasticity. The main difficulties in addressing this issue derive from its fully nonlinear framework, where no assumption of smallness of the deformation and displacement fields is formulated.

In [1] the displacement field of an inflexed solid has been defined by a kinematical model based on the following three assumptions: the solid is inflexed longitudinally with constant curvature; after deformation, cross sections maintain their planarity; also cross sections are inflexed transversely with constant 
curvature. This kinematic model has been modified in the present paper considering the class of slender beams, that is of solids whit the longitudinal dimension notably prevalent on the two transversal ones.

The displacement field has been obtained by examining the contributions due to the rigid translation, the rigid rotation and the pure deformation. Given the nonlinearity, these contributions have coupled expressions. For each point of the beam, the deformation gradient $\mathbf{F}$, the rotation tensor $\mathbf{R}$ and the stretch tensor $\mathbf{U}$ have been computed. Despite the elaborate shape of the displacement field, the stretches have simple and compact expressions in terms of exponential functions ( $c f$. eqn (24)). The deformation state is triaxial and non-homogeneous. Explicit formulae have been given to determine the area of cross sections and the volume of the beam in the deformed configuration.

Studied the kinematics of inflexed slender beams, a Lagrangian analysis of the equilibrium problem has been developed. Determined the Piola-Kirchhoff stress tensor $\mathbf{T}_{R}$ for a generic hyperelastic material, the equilibrium equations have been derived. These equations, which must be satisfied locally, have been expressed by a very complex system of three partial differential equations. Having adopted the semi-inverse approach, it is unthinkable that the above system of equilibrium equations can be correctly solved for all points of the beam. Nevertheless, a basic longitudinal line (with coordinates $X=Y=0$ and $Z=Z$ ) has been recognized, where the equilibrium equations are exactly satisfied.

At this point of the formulation, the stored energy function has been specified by assigning it the form of the compressible Mooney-Rivlin law. To assess the accuracy of the displacement field in correspondence of points different from those belonging to the basic line, by means of numerical analyses it has been estimated how much the equilibrium equations deviate from zero as one moves away from the basic line. By varying the geometrical and the constitutive parameters, the existence of a central core surrounding the basic line, where the equilibrium equations are close to zero, has been highlighted. The most important parameter is the length of the beam $L$, in the sense that the equilibrium equations are very close to zero at each point of the beam as the length $L$ grows. 
A further verification of the obtained solution has been performed by calculating

the normal force and checking that it is practically zero.

With the purpose of evaluating stretches and stresses in the deformed configuration, an Eulerian analysis has been conducted. The formulae allowing the transition from Lagrangian coordinates to Eulerian coordinates have been derived ( $c f$. eqn (26)). These has then been used to determine the diagrams of the stretches in the deformed cross sections ( $c f$. Figure 14). The diagrams of the stretches in the deformed configuration are linear. Also the neutral axis for the deformation (line $\lambda_{z}=1$ ) is rectilinear. All this according to the kinematic model, which predicts that cross sections remain plane and rotate rigidly around the neutral axis. This aspect of the problem could not been observed in terms of Lagrangian coordinates. In addition, the line $\lambda_{x}=\lambda_{y}=1$ is distinct from the neutral axis $\lambda_{z}=1$.

The Cauchy principal stresses has been evaluated ( $c f$. eqn (41) and the effective stress distributions in the inflexed beam are shown by some diagrams ( $c f$. Figures 16 and 17). Knowing the stress distributions, the bending moment in the deformed configuration has been determined ( $c f$. eqn 42 ), making it possible to assess the value of the moment needed to produce a specific inflexion angle $\alpha_{0}$. This has allowed to impose the boundary conditions statically through the application on the two end faces of the beam of a pair of self-equilibrated bending moments ( $c f$. Figure 19.

48 By imposing the hypothesis of smallness of the displacement and strain fields, the whole formulation exposed in the paper for the finite anticlastic bending 50 of hyperelastic slender beams has been linearized. All derived formulae have been rewritten as power series. These series, which depend on the radii $r$ and $R_{0}$, have been truncated by preserving the first order infinitesimals as $r \rightarrow \infty$ and $R_{0} \rightarrow \infty$. Accordingly, the nonlinear displacement field 22 has been linearized getting exactly the well-known displacement field of the linear theory of inflexed beams ( $c f$. eqn (48)). With the linearization of the deformation gradient, infinitesimal strain and infinitesimal rigid rotations tensors have been derived ( $c f$. eqn 52 and $(53)$ ). Due to the Poisson effect, the linearized strain 
state and stress state are triaxial and depend (linearly) only on the variable $Y$ and vanish for $Y=0$. Through linearization, it has been shown that, contrary to the finite theory, in the infinitesimal kinematics the height and the area of cross sections as well as the volume of the solid remain unchanged after deformation (cf. eqn (54)). The two stress measures of Piola-Kirchhoff and Cauchy in the linearized theory coincide. Therefore, linearizing the Piola-Kirchhoff tensor (15) and the Cauchy stress tensor $(39)$, the same stress tensor has been obtained ( $c f$. eqn (55), (56) and (57)).

Differently from the bending of nonlinear beams, in the infinitesimal theory the neutral axis of strain coincides with the neutral line of the stress and they pass through the centroid of the cross section.

Since different constitutive laws have been used, the stresses obtained with the linearization are not equal to the typical stresses of an inflexed beam in infinitesimal theory. Therefore, the relationships that must be met the constitutive parameters of a compressible Mooney-Rivlin material in order to reproduce the Lamé constants have been identified ( $c f$. eqn (62)). Using these relationships, the linearized stress tensor coincides with that typical of the infinitesimal theory. In particular, the state of stress becomes uniaxial.

The linearization procedure has demonstrated the complete transition from the proposed solution for the fully nonlinear bending to the classical solution for the infinitesimal bending of beams.

In order to corroborate the theoretical model about the inflexion of slender beams in a fully nonlinear context, a numerical model and an experimental investigation have been developed. The main results provided by these two different approaches have been then compared with those provided by the theoretical model formulated in the Section 2.4

The FEM model has been used to perform a numerical analysis. The beam has been partitioned using the 4 node tetrahedra elements. Difficulties have been encountered to impose the boundary conditions, which prescribe the rotations of the two end cross sections of the beam. As a consequence, the obtained results near the terminal portions of the beam has appeared less accurate. 
For a neoprene filled rubber, the constitutive parameters of the compressible Mooney-Rivlin stored energy function have been experimentally identified [32. Therefore, a sample with the shape of a slender prismatic beam has been made with this rubber-like material. The sample has been bent by imposing large rotations to its end cross sections through a mechanical equipment prototype, which has been properly designed and manufactured to perform the experimental analysis. In particular, the beam-like sample has been subjected to large bending by using two pantographs. The DIC instrumentation has been used to acquire experimental data. Such an equipment allows to measure and monitor the entire displacement field of the specimen external surface with great accuracy $( \pm 10 \mu m)$.

Based on three different approaches (theoretical model, numerical simulation and experimental investigation), the following results for the nonlinear equilibrium problem at hand have been found.

In the evaluation of the vertical displacement field (assessed on both the lateral and upper surfaces of the sample), the three different approaches have yielded almost coincident results. The sample has been bent longitudinally with constant curvature, and the same radius of curvature has been evaluated with the three methods varying the angle of rotation $\alpha_{0}$. This fact has corroborated the first kinematic hypothesis formulated in the first part of this paper. Moreover, owing to the anticlastic effect, also the cross sections have exhibited inflexion in their planes. With the exception of the experimental results found for $\alpha_{0}$ small, the transversal inflexion has occurred with constant curvature and almost the same radius of curvature has been provided by the three different approaches varying $\alpha_{0}$. This has confirmed the third kinematic hypothesis.

Since the displacement field inside the specimen cannot be measured through the DIC system, for the internal points of the sample the results obtained from the theoretical model have been compared with those provided by the numerical analysis only, founding good agreement. Concerning the displacements of the longitudinal and transverse basic lines, the two models have provided coincident results, thus confirming the validity of the first and third hypotheses. Very 
similar results obtained with these two approaches have been found also for the rotation field of the cross sections, as well as for the stretches and stresses, which have been evaluated both in the Lagrangian and in the Eulerian configurations.

The linear trend of the longitudinal stretches along the depth of the cross section in the Eulerian configuration, evaluated through the FE code, has corroborated the second kinematic hypothesis of the theoretical model about the preservation of the planarity of the cross sections after the deformation.

\section{Acknowledgement}

Financial support from the Italian Ministry of Education, University and Research (MIUR) in the framework of the Project PRIN "Modelling of constitutive laws for traditional and innovative building materials" (code 2017HFPKZY) is gratefully acknowledged.

[1] L. Lanzoni, A. M. Tarantino, Finite anticlastic bending of hyperelastic solids and beams, Journal of Elasticity 131 (2) (2018) 137-170.

[2] B. Seth, Finite strain in elastic problems, Philosophical Transactions of the Royal Society of London. Series A, Mathematical and Physical Sciences 234 (738) (1935) 231-264.

[3] R. Rivlin, Large elastic deformations of isotropic materials. V. the problem of flexure, Proceedings of the Royal Society of London. Series A. Mathematical and Physical Sciences 195 (1043) (1949) 463-473.

[4] J. L. Ericksen, Deformations possible in every isotropic, incompressible, perfectly elastic body, Zeitschrift für angewandte Mathematik und Physik (ZAMP) 5 (6) (1954) 466-489.

[5] M. M. Carroll, Finite deformations of incompressible simple solids i. isotropic solids, The Quarterly Journal of Mechanics and Applied Mathematics 21 (2) (1968) 147-170. 
[6] C. C. Wang, Normal configurations and the nonlinear elastostatic problems of bending, torsion, expansion, and eversion for compressible bodies, Archive for Rational Mechanics and Analysis 114 (3) (1991) 195-236.

[7] M. Aron, Y. Wang, On deformations with constant modified stretches describing the bending of rectangular blocks, The Quarterly Journal of Mechanics and Applied Mathematics 48 (3) (1995) 375-387.

[8] G. Saccomandi, Universal results in finite elasticity, non-linear elasticity: theory and applications, London Mathematical Society lecture notes 283 (2001) 97-134.

[9] R. Shield, Bending of a beam or wide strip, The Quarterly Journal of Mechanics and Applied Mathematics 45 (4) (1992) 567-573.

[10] J. Blondin, Horace Lamb. Sur la flexion d'un ressort élastique plat; Phil. Mag., 5e série, t. XXXI, p. 182-188; 1891, J. Phys. Theor. Appl. 1 (1) (1892) 182-188.

[11] A. E. H. Love, A treatise on the mathematical theory of elasticity, Cambridge university press, 2013.

[12] T. Wang, S. Lee, O. Zienkiewicz, A numerical analysis of large deflections of beams, International Journal of Mechanical Sciences 3 (3) (1961) 219-228.

[13] T. Wang, Non-linear bending of beams with uniformly distributed loads, International Journal of Non-Linear Mechanics 4 (4) (1969) 389-395.

[14] J. Holden, On the finite deflections of thin beams, International Journal of Solids and Structures 8 (8) (1972) 1051-1055.

[15] R. Frisch-Fay, Flexible bars, Butterworths, 1962.

[16] E. Reissner, On one-dimensional finite-strain beam theory: the plane problem, Zeitschrift für angewandte Mathematik und Physik ZAMP 23 (5) (1972) 795-804. 
[17] E. Reissner, On one-dimensional large-displacement finite-strain beam theory, Studies in applied mathematics 52 (2) (1973) 87-95.

[18] E. Reissner, On finite deformations of space-curved beams, Zeitschrift für angewandte Mathematik und Physik ZAMP 32 (6) (1981) 734-744.

[19] K. J. Bathe, S. Bolourchi, Large displacement analysis of three-dimensional beam structures, International Journal for Numerical Methods in Engineering 14 (7) (1979) 961-986.

[20] J. C. Simo, A finite strain beam formulation. the three-dimensional dynamic problem. part i, Computer methods in applied mechanics and engineering 49 (1) (1985) 55-70.

[21] A. Cardona, M. Géradin, A beam finite element non-linear theory with finite rotations, International journal for numerical methods in engineering 26 (11) (1988) 2403-2438.

[22] J. C. Simo, L. Vu-Quoc, On the dynamics in space of rods undergoing large motionsa geometrically exact approach, Computer methods in applied mechanics and engineering 66 (2) (1988) 125-161.

[23] A. Ibrahimbegović, On finite element implementation of geometrically nonlinear reissner's beam theory: three-dimensional curved beam elements, Computer methods in applied mechanics and engineering 122 (1-2) (1995) $11-26$.

[24] P. G. Ciarlet, G. Geymonat, Sur les lois de comportement en élasticité non linéaire compressible, CR Acad. Sci. Paris Sér. II 295 (1982) 423-426.

[25] M. Destrade, G. Saccomandi, I. Sgura, Methodical fitting for mathematical models of rubber-like materials, Proceedings of the Royal Society A: Mathematical, Physical and Engineering Sciences 473 (2198) (2017) 20160811.

[26] A. M. Tarantino, Homogeneous equilibrium configurations of a hyperelastic compressible cube under equitriaxial dead-load tractions, Journal of Elasticity $92(2008) 227-254$. 
1100

1102

1104

[27] A. M. Tarantino, Equilibrium paths of a hyperelastic body under progressive damage, Journal of Elasticity 114 (2) (2014) 225-250.

[28] L. Lanzoni, A. M. Tarantino, Equilibrium configurations and stability of a damaged body under uniaxial tractions, Zeitschrift für angewandte Mathematik und Physik 66 (1) (2015) 171-190.

[29] L. Lanzoni, A. M. Tarantino, A simple nonlinear model to simulate the localized necking and neck propagation, International Journal of Non-Linear Mechanics 84 (2016) 94-104.

[30] F. Falope, L. Lanzoni, A. Tarantino, Bending device and anticlastic surface measurement of solids under large deformations and displacements, Mechanics Research Communications 97 (2019) 52-56.

[31] L. Lanzoni, A. M. Tarantino, Damaged hyperelastic membranes, International Journal of Non-Linear Mechanics 60 (2014) 9-22.

[32] A. M. Tarantino, L. Lanzoni, F. O. Falope, The bending theory of fully nonlinear beams, Springer, 2019. 